\title{
Magnetic Stimulation of Neural Tissue: Techniques and System Design
}

\author{
Eric Basham, Zhi Yang, Natalia Tchemodanov, and Wentai Liu
}

\begin{abstract}
Magnetic stimulation of neural tissue is an attractive technology because neural excitation may be affected without the implantation of electrodes. This chapter provides a brief overview of the technology and relevant literature. While extensive magnetic stimulation modeling and clinical experimentation work has been presented, considerably less quantitative in vitro work has been performed. In vitro experiments are critical for characterizing the site of action, the structures stimulated, and the long-term tissue histological effects. In vitro systems may also facilitate the development of novel magnetic stimulation approaches. To demystify magnetic stimulation systems, this chapter presents an in vitro experimental system using a systematic design methodology. The modeling methods are designed to aid experimentation. Circuit schematics, test rigs, and supplier information are given to support practical implementation of this design methodology. Example neural preparations and their modeling and use are also covered. Finally, as an alternative to pulsed discharge circuits for magnetic stimulation, this chapter shows how to use a circuit to deliver asymmetric current pulses to generate the magnetic field.
\end{abstract}

\section{Introduction}

Magnetic stimulation of neural tissue is an intriguing technology because stimulation may be affected without direct contact to the tissue under study. Magnetic stimulation has advantages over electrical stimulation in biocompatibility, bioresistance, and operational biotoxicity. Rather than creating an electric field via the injection of current, as in electrical stimulation, magnetic stimulation arises as a result of the induced fields created within the tissue. A conceptualized system is shown in Fig. 1. In short, a time-varying current flowing through a coil generates a time-varying magnetic field. The time-varying magnetic field induces an electrical field within

\footnotetext{
E. Basham $(\bowtie)$

Department of Electrical Engineering, University of California, Santa Cruz, CA, USA

e-mail: basham.eric@gmail.com
} 


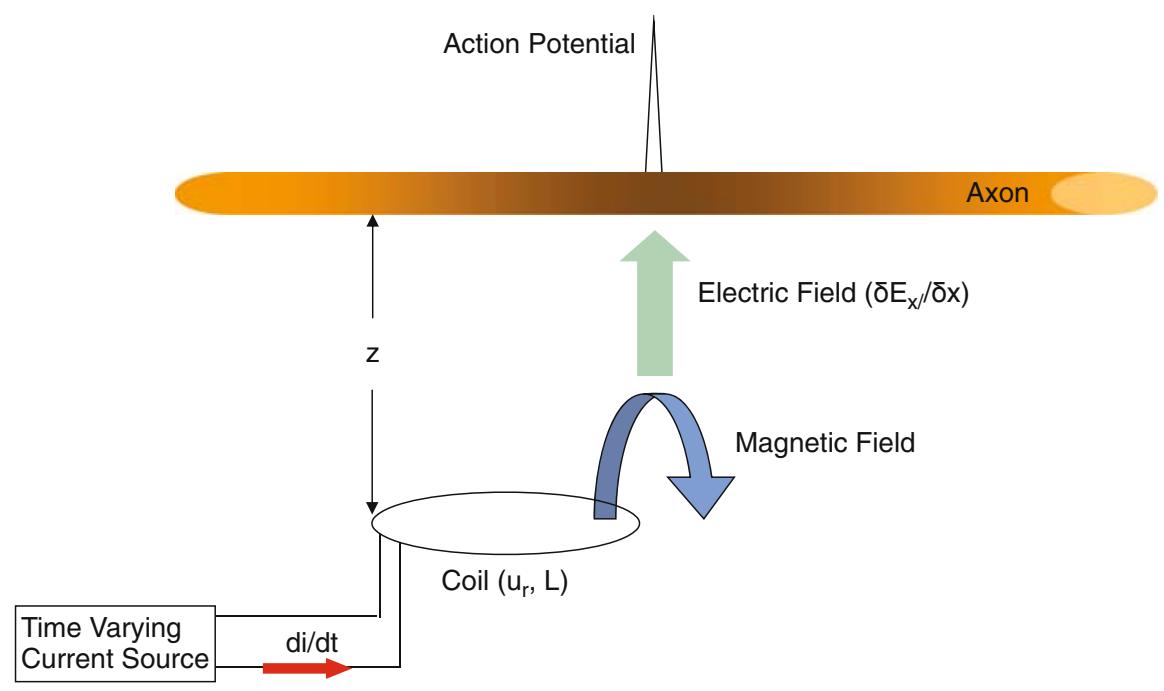

Fig. 1 Magnetic stimulation conceptual system overview. Critical system design parameters are the current ramp rate of change di/dt, the coil relative permeability $\mathrm{u}_{\mathrm{r}}$, inductance $\mathrm{L}$, and the coil distance from the nerve $\mathrm{z}$. The spatially varying electric field $\partial \mathrm{E}_{\mathrm{x}} / \partial \mathrm{x}$ produced depolarizes the axon, shown as the shaded area along the axon, and leads to the initiation of an action potential

the tissue. During magnetic stimulation, the stimulating coil may be sealed and remain completely isolated from the target tissue. Since there is no metal-electrolyte interface, as is the case with electrodes used for electrical stimulation, issues of charge transfer, electrode surface modification, and corrosion are mitigated. In addition, magnetic fields penetrate unattenuated through non-conductive tissue because the permeability of tissue at low frequencies $(<50 \mathrm{kHz})$ is near unity.

Since no direct electrical connection between the target tissue and the device is required, magnetic stimulation applied externally may be used to stimulate neural tissue without surgical implantation of electrodes. One of the most important applications of external excitation of neural tissue is transcranial magnetic stimulation (TMS). TMS is used for mapping functional areas of the brain, sleep studies, and the treatment of depression. External magnetic stimulation has also been used as an alternative to functional electrical stimulation (FES) to mitigate incontinence, treat pain [1], evaluate spinal function, and as a diagnostic tool for evaluation of nerve damage [2]. Reviews of magnetic stimulation and TMS in particular are available in [3-8].

Magnetic stimulation with traditional methods confounds in vitro experimentation as the area of effect is quite large and interferes with standard electrophysiology recording equipment. The resulting electromagnetic interference and mechanical constraints are especially evident in adherent cell electrophysiological studies of magnetic stimulation. Typically, only large and long neural preparations (centimeters in length) can be used for experimentation. A properly scaled system also facilitates the study of the histological effects of magnetic stimulation 
and aids in the investigation of pulsed electromagnetic fields on nerve regrowth. These are both active areas of investigation [9-11]. Currently, commercially available systems or system descriptions are lacking for important neuroscience animal models such as rat or mouse. Scaled magnetic stimulation systems may also facilitate animal model experimentation and lead to insight not available through clinical experimentation.

While extensive magnetic stimulation modeling work has been presented [12-17], considerably less quantitative in vitro work has been performed [18-20]. In vitro experiments are critical for characterizing the site of action, structures stimulated, and the long-term tissue histological effects of magnetic stimulation. The disparity in experimental versus modeling research is predominantly due to the difficulty in understanding the electromagnetic interaction with neural tissue during experimentation. For example, inserting electric probes into the tissue during stimulation alters the fields produced and thus the experimental results.

There is still some debate about the exact nature of the interaction of magnetic fields with nervous tissue [21-23], exemplifying the need for flexible, quantitative experimental systems. In a recent clinical evaluation of repetitive TMS (rTMS), the key difference in efficacy was found to link closely with the manufacturer model (and thus the specific waveform) generating the biphasic pulse [24].

Modifying full-scale magnetic stimulation systems in a clinical environment presents significant challenges to proof of concept experimentation. As an example, there is little data that covers co-stimulation (pairing electrical stimulus with magnetic stimulus) and paired pulse protocols. Rapidly reconfigurable in vitro systems can be invaluable for developing better predictive models, correlating effects of clinical experiments [25], and developing proof of concept systems.

In the following chapter, we outline a method that relies on the use of first-order estimates to facilitate experimentation. We hope to demystify magnetic stimulation experiments by simplifying the modeling methods and describing the experimental apparatus. Since magnetic stimulation systems are more complex than electrical stimulation systems, both from the perspective of modeling and apparatus, we touch on design techniques for the modeling, computer control, data acquisition, coil design, circuit design, and recording methods. We then use a scaled magnetic stimulation system to investigate a new approach to magnetic stimulation circuit design. Two example neurological preparations and the reasoning for their use are also covered. While at some points the discussion may appear oversimplified or pedantic, the focus of this chapter is to facilitate lab experimentation and offer a conceptual starting point for further experimentation.

\section{Field-Based Comparison of Electrical and Magnetic Stimulation}

Compared to magnetic stimulation of neural tissue, electrical stimulation is well understood and well characterized. In 1947, Hodgkin and Rushton reported the first experimental application of passive cable theory to axons [26]. A significant review of experimental results of extracellular electrical stimulation later 
occurred in [27], and the author presented an instructional chapter oriented toward laboratory practice and qualitative analysis in [28]. In [29], Rattay introduced an efficient quantitative approach for modeling the response of an axon to extracellular stimulation. The method introduced the concept of the "activation function" for prediction of extracellular stimulation response. For extracellular electrical stimulation, the second derivative of the external potential in the direction of the axon is responsible for activation of the axon. Rattay's work focused on the derivation of a modified form of the cable equation that included a source term shown on the left-hand side of Equation (1)

$$
\lambda_{m}^{2} \frac{\partial^{2} V_{e}(x, t)}{\partial x^{2}}=-\lambda_{m}^{2} \frac{\partial^{2} V_{m}(x, t)}{\partial x^{2}}+\tau \frac{\partial V_{m}(x, t)}{\partial t}+V_{m}(x, t),
$$

where the length and time constants of the neural membrane $\left(\lambda_{\mathrm{m}}\right.$ and $\tau_{\mathrm{m}}$, respectively) are defined as

$$
\lambda_{\mathrm{m}}=\sqrt{\frac{r_{m}}{r_{i}}} \text { and } \tau_{\mathrm{m}}=\mathrm{c}_{\mathrm{m}} \mathrm{r}_{\mathrm{m}}
$$

$\mathrm{V}_{\mathrm{m}}$ is the transmembrane voltage defined as the voltage difference between the intracellular and the extracellular fluid $\left(\mathrm{V}_{\mathrm{m}}=\mathrm{V}_{\text {intracellular }}-\mathrm{V}_{\text {extracellular }}\right), \mathrm{r}_{\mathrm{m}}$ is membrane resistance times unit length $\left(\mathrm{k} \Omega \cdot \mathrm{cm}\right.$ axon length), $\mathrm{r}_{\mathrm{i}}$ is intracellular resistance $\left(\Omega \cdot \mathrm{cm}^{-1}\right), \mathrm{c}_{\mathrm{m}}$ is membrane capacitance per unit length $\left(\mathrm{F} \cdot \mathrm{cm}^{-1}\right) . \mathrm{V}_{\mathrm{e}}$ is the stimulating electrode voltage and the axon lies along the $\mathrm{x}$ axis as depicted in Fig. 2. Both $V_{e}$ and $V_{m}$ are functions of location and time. Setting $V_{m}$ to zero leads to the definition of the "activating function", $\lambda^{2} \cdot \partial \mathrm{E}_{\mathrm{e}} / \partial \mathrm{x}$, which is useful for determining the initial change in $V_{\mathrm{m}}$.

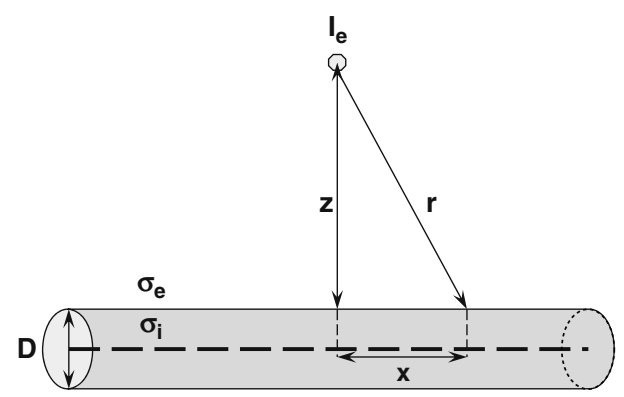

Fig. 2 Geometric description of stimulation of a long uniform fiber by a point source. An extracellular point current source $I_{e}$ is located a distance $z$ from the cylindrical fiber of diameter $\mathrm{D}$ and intracellular conductivity $\sigma_{\mathrm{i}}$. Fiber and source are immersed into an unbound extracellular medium of uniform conductivity $\sigma_{\mathrm{e}}$. The geometrical representation is used to derive the electrical field distribution along the fiber

The activating function allows estimation of stimulation based on the membrane length constant and the spatially varying electric field [30]. The activation function provides a method to quantitatively predict the behavior of the axon to external electrical fields. Regions of the cable where $\partial^{2} \mathrm{~V}_{\mathrm{e}} / \partial \mathrm{x}^{2}$ 
(a)

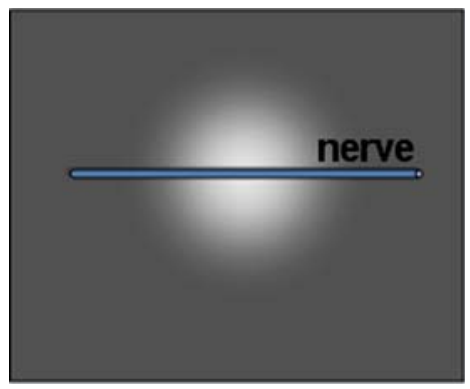

(b)

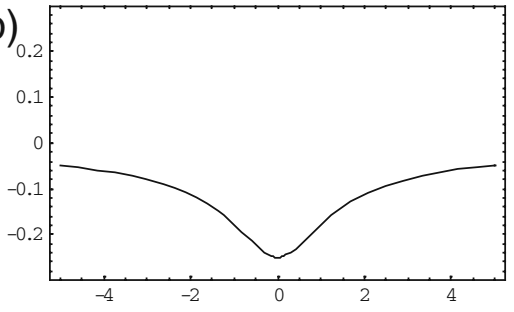

(c)

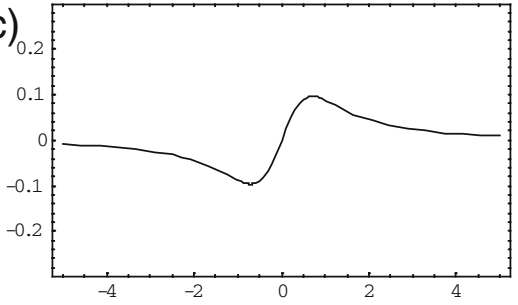

(d)

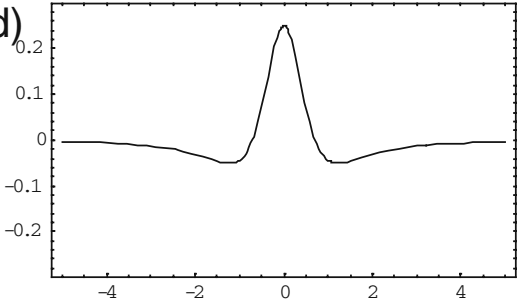

(e)

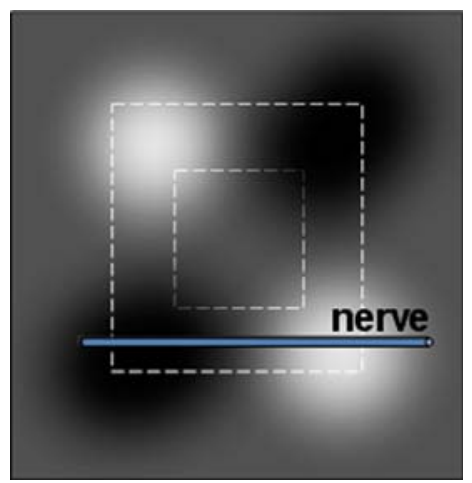

(f)

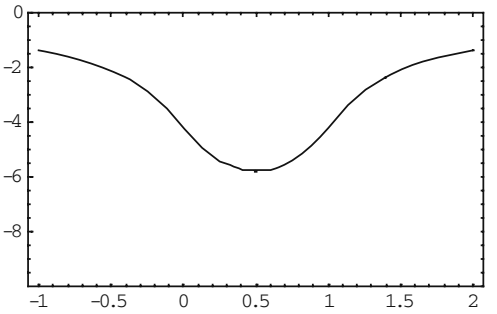

(g)

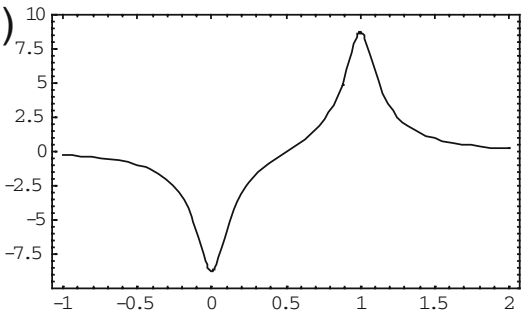

Fig. 3 Diagrammatic comparison of fields induced by a cathodal point source (a-d) and a square magnetic stimulation coil (e- $\mathbf{g})$. (a) nerve lies above the point source and the shaded area represents the magnitude of the spatially varying electric field. With the nerve fiber placed as shown in (a), (b) shows the magnitude of the extracellular potential $\mathrm{V}_{\mathrm{e}}$ along the fiber, (c) shows the magnitude of the electric field along the fiber, and (d) shows the derivative of electric field along the fiber for cathodal electrical stimulation. (e) nerve lies above the coil and the shaded area represents the positive magnitude (light shading) and negative magnitude (dark shading) of the spatially varying electric field. With the nerve fiber placed as shown in (e), (f) shows the magnitude of the electric field along the fiber, and (g) shows the derivative of electric field along the fiber for magnetic stimulation. Comparing the two methods, cathodal electrical stimulation generates a single depolarizing peak and two much smaller hyperpolarizing peaks, while magnetic stimulation generates a hyperpolarizing and a depolarizing peak of approximately equal magnitude. As a note: the situation for anodic electrical stimulation varies slightly as a strong hyperpolarizing peak and two small depolarizing peaks are generated. Excitation occurs when the small depolarizing peaks reach the necessary magnitude to fully depolarize the fiber. Typically 5-7 times as much current are required to stimulate anodically as cathodically 
were greater than zero were shown to be depolarized. Regions where $\partial^{2} V_{e} / \partial x^{2}$ are less than zero are predicted to be hyperpolarized, as depicted in Fig. 3.

$$
V_{e}=\frac{I_{e}}{4 \pi \sigma\left(x^{2}+z^{2}\right)}
$$

The electric field is equal to the gradient of the scalar potential, $V_{\mathrm{e}}$, from the point source electrode

$$
\vec{E}_{e}=\frac{\partial V_{e}}{\partial x}=\frac{I_{e} x}{4 \pi \sigma\left(x^{2}+z^{2}\right)^{\frac{3}{2}}}
$$

where $I_{e}$ is the current injected by the point electrode, $\sigma$ is the extracellular medium conductivity, and $\mathrm{z}$ is the distance between the point source and the axon. The spatially varying electric field from a point source electrode at a height, $\mathrm{z}$, above the axon is

$$
\frac{\partial \vec{E}_{e}}{\partial x}=\frac{I_{e}\left(2 x^{2}-z^{2}\right)}{4 \pi \sigma\left(x^{2}+z^{2}\right)^{\frac{5}{2}}} .
$$

Thus, we can directly calculate $\delta \mathrm{E}_{\mathrm{e}} / \delta \mathrm{x}$ from Equation (5) given the electrode input current, extracellular conductivity, position of the electrode, and the cable equation. With the source term modified to reflect the spatially varying electric field the cable equation is

$$
\lambda^{2} \frac{I_{e}\left(2 x^{2}-z^{2}\right)}{4 \pi \sigma\left(x^{2}+z^{2}\right)^{\frac{5}{2}}}=\lambda^{2} \frac{\partial \vec{E}_{e}}{\partial x}=-\lambda_{m}^{2} \frac{\partial^{2} V_{m}(x, t)}{\partial x^{2}}+\tau \frac{\partial V_{m}(x, t)}{\partial t}+V_{m}(x, t) .
$$

There is some debate in the literature as to whether the activation function is more conveniently defined as $\partial \mathrm{E}_{\mathrm{e}} / \partial \mathrm{x}$ or as $\lambda^{2} \cdot \partial \mathrm{E}_{\mathrm{e}} / \partial \mathrm{x}[31]$, but here we use activating function to refer to $\lambda^{2} \cdot \partial \mathrm{E}_{\mathrm{e}} / \partial \mathrm{x}$, and define the spatially varying electric field as $\delta \mathrm{E}_{\mathrm{e}} / \delta \mathrm{x}$, that is, the rate of change of the electric field with respect to the $x$ axis.

The cable model provides insight into the interaction between the electric fields and the neural tissue, but it does not completely describe the dynamics of the system. The peak spatially varying electric field and the length of time the electric field is maintained are both essential components to understanding the generation of an action potential in neural tissue. For example, the activating function fails to correctly predict the depolarized or hyperpolarized regions for longer time periods or wider areas of stimulation (p. 214, [32]). Vexing questions about the validity of the activation function remain [33, 34]. These inconsistencies have led to efforts to improve the passive cable model's predictive capability via simulation [23, 35-40].

Several time points should be collected to facilitate the correlation of modeling and experimental data. The plot of time point versus stimulus strength is called a strength-duration curve. The strength-duration curve is an invaluable experimental metric. Analysis of experimental data can be used to obtain an estimate of $\tau_{\mathrm{m}}$ through fitting of Equation (7). The curves can also be qualitatively compared and the comparison can provide some insight into physiological conditions. 


$$
I_{t h}=\frac{I_{\text {rheobase }}}{\left(1-e^{-\left(\frac{t_{\text {duration }}}{\tau}\right)}\right)} .
$$

In this equation, $\mathrm{I}_{\mathrm{th}}$ is the current stimulus threshold sufficient to generate a neural response (action potential) in $50 \%$ of the trials. $t_{\text {duration }}$ is the length of the experimental stimulus pulse, and $\tau_{\mathrm{m}}$ is the membrane time constant as defined in Equation (2). $\mathrm{I}_{\text {rheobase }}$ is the minimum current stimulus that produces any response as $t \rightarrow \infty$. Chronaxy is defined as the duration at which stimulus occurs at twice the rheobase. During actual experimentation, the rheobase is estimated at approximately $10 \times$ the chronaxy of the nerve. More information on the derivation and application of the strength-duration curve may be found in [41].

Using $I_{t h}$ as the dependant variable of the strength-duration curve for characterization of nerves and axons discards important information about the actual mechanism of excitation. An improved method is the use of $\delta \mathrm{E}_{\mathrm{e}} / \delta \mathrm{x}$, as derived in Equation (5). Further verification is possible by measuring strength-duration curves at several distances above the nerve. However, the range at which stimulus will occur at any input current will vary as a function of the membrane constants, pulse polarity, and diameter of the axon as shown in Fig. 4.

Using $\delta \mathrm{E}_{\mathrm{e}} / \delta \mathrm{x}$ as the ordinate of a strength-duration curve allows the comparison of electric fields generated by sources other than a point source electrode. For example, an equivalent cable expression to the cable equation with an electrical source term (Equation 8) for magnetic stimulation was presented in [15]. The spatially varying electric field was derived from fundamental electromagnetic principles.

$$
\lambda_{m}^{2} \frac{\partial \vec{E}_{x}(x, t)}{\partial x}=-\lambda_{m}^{2} \frac{\partial^{2} V_{m}(x, t)}{\partial x^{2}}+\tau \frac{\partial V_{m}(x, t)}{\partial t}+V_{m}(x, t)
$$

where $\mathrm{E}_{\mathrm{x}}$ is the $x$ component of the magnetically induced electric field. The derivation of (Equation 8) assumes the fiber lies along the $x$ axis parallel to the plane of the coil. Early reports of magnetic stimulation used a magnetic field strength term to report strength-duration curves (e.g., [42]). However, comparing the values of electrically and magnetically induced spatially varying electric fields $\left(\delta \mathrm{E}_{\mathrm{e}} / \delta \mathrm{x}\right.$ and $\left.\delta \mathrm{E}_{\mathrm{x}} / \delta \mathrm{x}\right)$ allows a first-order comparison between magnetic and electrical stimulation.

There is still some debate as to whether the magnetically induced $\delta \mathrm{E}_{\mathrm{x}} / \delta \mathrm{x}$ is applicable to neural stimulation [43]. A controlled, quantitative, experimental approach allows the investigation of conflicting clinical and experimental evidence and should provide insight into the discrepancy between simulation and experimental data. Cable equation modeling seems to lend itself to 


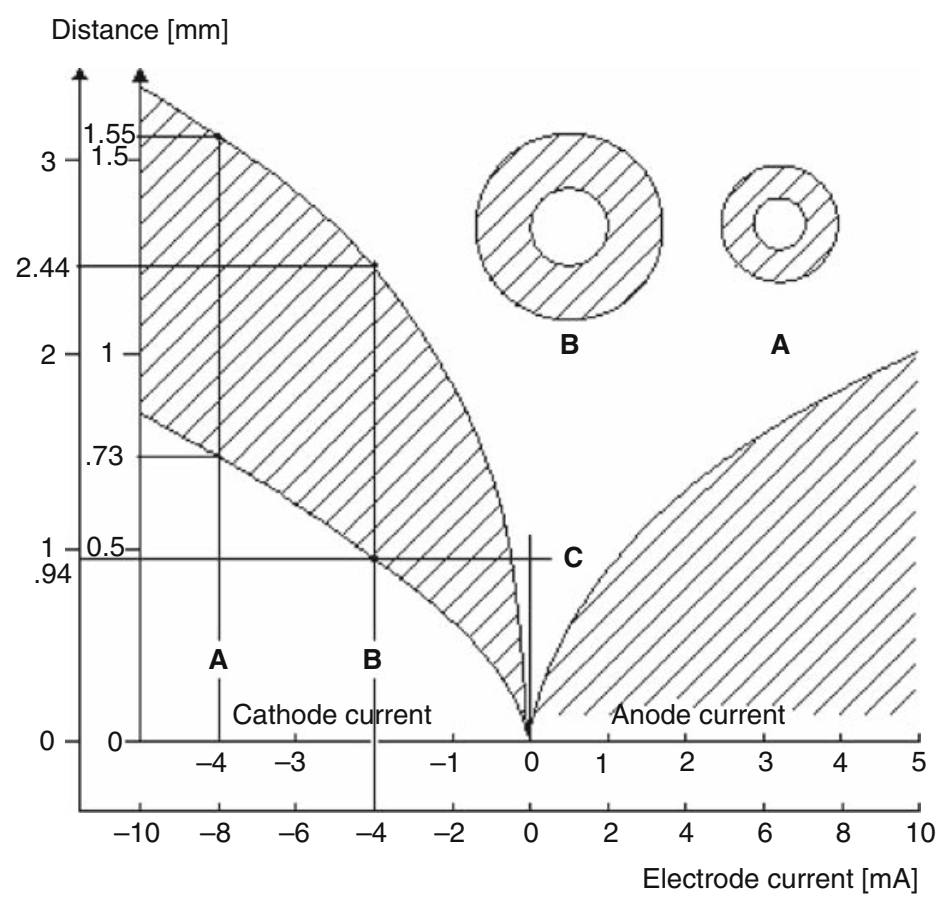

Fig. 4 Current-distance relationship for unmyelinated fibers. Excitation occurs for points lying in the shaded region. For cathodal stimulation, a minimum distance arises at the point where anodal block prevents the escape of the action impulse. For anodal stimulation, block does not occur; thus there is no lower limit on the source-fiber distance. The inner scales are for a fiber diameter of $9.6 \mu \mathrm{m}$, and the outer for a diameter of $38.4 \mu \mathrm{m}$. Scaling the excitation with respect to both current strength, source-fiber distance, and fiber diameter leaves the solution unchanged (from [29])

certain neurological preparations, notably long, relatively uniform nerve fibers. Scaling a magnetic stimulation system to allow the use of smaller and more varied preparations with varying axonal diameter and membrane constants can further facilitate modeling. Several candidate neural preparations are well characterized and include information on the membrane constants and the diameter of individual axons. For dynamic modeling, the active properties of the membrane must be solved or simulated [15, 44]. Using well-characterized preparations allows simulation of the cable equation and several freely available packages are designed to assist with cable modeling, including NEURON (http://www.neuron.yale.edu/neuron/) [45] and GENESIS (http://www.genesis-sim.org/GENESIS/) [46]. Neurocal is a simplified package written in MATLAB that is very easy to use and modify [47]. Combining easily modeled, well-documented neurological preparations with simple lab experiments and accurate, easy to use active cable equation simulators is a powerful application of the engineering methodology of simulate, design, fabricate, and test. 


\section{Magnetic Modeling}

There are several different approaches to determining the source term in the cable equation for magnetic stimulation. In any case, the goal is the same: determine the unknown experiential variables from the known experimental parameters. For magnetic stimulation, the unknown terms are the induced electrical field and the spatial variation of the induced electric field, while the design parameters are the time-varying input current, coil shape, turn number, and expected depth of stimulation. The required excitation threshold may be determined using methods outlined in the prior section, but to some degree may also be considered an experimental variable. In the final section, we will cover selection of neural preparations with widely varying membrane properties which are useful for testing the same experimental set-up with neural preparations having differing excitability thresholds.

Finite Element Methods (FEM) can provide answers directly by solving the fundamental electromagnetics problems at each point of a finely discretized physical model. Ansoft, COMSOL, Quickfield, and MATLAB are finite element analysis software packages that have been applied to magnetic stimulation modeling. All of these packages also have a free or low-cost student version. Texts that introduce the software concurrently with electromagnetic principles include for Ansoft [48], for Quickfield and MATLAB [49], and for MATLAB specifically [50]. Magnetic stimulation modeling becomes complex quickly as realistic models are developed. These packages also have a significant learning curve to master and apply appropriately. In addition, finite element models can be computationally time consuming to evaluate. These reasons make finite element methods applied to magnetic stimulation more appropriate for design verification than design.

Analytical solutions address these concerns. Derivation of the fields involved in magnetic stimulation can be daunting for those less experienced with electromagnetics. In many reviews of magnetic stimulation the authors skim the general approach and leave the reader without the tools necessary to aid experimentation. We outline the general approach to the derivation of the fields involved in magnetic stimulation and subsequently provide the reader with analytical formulas that can provide first-order estimates of the source term in the passive cable model equation. For readers with advanced electromagnetic understanding, there are alternate approaches to determining the relevant electrical fields. We have provided one example in the appendix covering scaling and the use of ferrite solenoid cores.

While the goal is to model the induced electric field $\vec{E}$ directly from the input current and physical coil parameters, the magnetic vector potential $\vec{A}$ is a more convenient way of deriving the induced electric fields. While the electric field, $\vec{E}$, can be described as the gradient of the scalar potential V (as outlined in the section above on cable modeling), there is no generalized scalar potential for magnetic field. However, the magnetic field, $\vec{B}$, can be obtained by taking the curl of the magnetic vector potential, $\vec{A}$.

$$
\vec{B}=\nabla \times \vec{A}
$$


The electric field, $\mathbf{E}$, is related to the time-varying magnetic field by

$$
\nabla \times \vec{E}=-\frac{\partial \vec{B}}{\partial t}
$$

Substituting and solving, we obtain

$$
\vec{E}=-\frac{\partial \vec{A}}{\partial t}-\nabla V
$$

Since there is assumed to be no charge on the coil and the current distribution in the coil is determined to be uniform (i.e. quasistatic conditions), Equation (11) reduces to

$$
\vec{E}=-\frac{\partial \vec{A}}{\partial t}
$$

The magnetic vector potential is related to the physical dimensions of the coil according to Fig. 5.

$$
\vec{A}=\frac{\mu_{0} N\left(\frac{d i}{d t}\right)}{4 \pi} \cdot \oint \frac{d l}{r}
$$

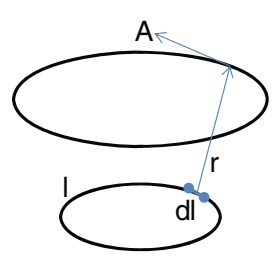

Fig. 5 Geometrical derivation of the magnetic vector potential A. The lower loop is the coil and the upper loop the region around which the magnetic vector potential is calculated

Much of the challenge in determining the electrical field induced by timevarying magnetic fields arises in accounting for the charge accumulation at the boundary interface between air and tissue. Several articles are available that address boundary condition modeling [51-56]. The interested reader is referred to them for more information. In the method presented in [57], for experimental conditions where the tissue interface is parallel to the plane of the coil, the electric field $\vec{E}_{x}$ can be calculated by numerically integrating along the line:

$$
\partial \vec{E}_{x}=-\frac{\mu_{0} N\left(\frac{d i}{d t}\right)}{4 \pi}\left\{\left[\frac{d l_{x}}{R}+\frac{\left(x-x_{0}\right) d l_{z}}{\rho^{2}} \cdot\left(1-\frac{z_{0}-z}{R}\right)\right] \hat{x}+\left[\frac{d l_{y}}{R}+\frac{\left(y-y_{0}\right) d l_{z}}{\rho^{2}}\left(1-\frac{z_{o}-z}{R}\right)\right] \hat{y}\right\}
$$

where the coil element lies at $\left(x_{0}, y_{0}, z_{0}\right)$, the electric field is calculated at $(x, y, z)$, $N$ is the number of coil turns, di/dt is the rate of change of the coil input current, $\mu_{0}$ is the permittivity of free space and

$$
\rho=\sqrt{\left(x-x_{0}\right)^{2}+\left(y-y_{0}\right)^{2}}
$$




$$
R=\sqrt{\left(x-x_{0}\right)^{2}+\left(y-y_{0}\right)^{2}+\left(z-z_{0}\right)^{2}} .
$$

The problem can be significantly simplified if the coil is planar and square. If the coil is in the $x-y$ plane with $z_{\mathrm{O}}=0$, and if no coil element lies in the $z$ axis, that is, all coil elements are parallel to the air-tissue interface, then Equation (13) simplifies to Equation (16). We omit the derivations of $\vec{E}_{y}$ and $\delta \mathrm{E}_{\mathrm{y}} / \delta \mathrm{y}$ because they are similar in form. While all elements in a square coil are parallel and assumed to be tightly packed, the width of the coil has finite dimensions with respect to the coil diameter, even more so for coils with small diameter. These assumptions introduce error, in some cases significant, but suffice for derivation of first-order design equations.

$$
\partial \vec{E}_{x}=-\frac{\mu_{0} N\left(\frac{d i}{d t}\right)}{4 \pi} \cdot \frac{1}{R} d l_{x}
$$

Integrating the expression with respect the $x$ component of the line yields the general form

$$
\vec{E}_{x}=-\left.\frac{\mu_{0} N\left(\frac{d i}{d t}\right)}{4 \pi} \cdot \ln \left[x-x_{0}+\sqrt{\left(x-x_{0}\right)^{2}+\left(y-y_{0}\right)^{2}+\left(z-z_{0}\right)^{2}}\right]\right|_{x_{01}} ^{x_{02}}
$$

A square coil has a side length of (a) and is oriented such that one corner of the coil is at $(0,0)$ and the opposite corner is at $(a, a)$. Two elements of the coil lie along the $x$ axis. The first element starts at $(0,0)$ and ends at $(a, 0)$. The expression is evaluated from 0 to $a$ as

$$
\vec{E}_{x}=-\left.\frac{\mu_{0} N\left(\frac{d i}{d t}\right)}{4 \pi} \cdot \ln \left[x-x_{0}+\sqrt{\left(x-x_{0}\right)^{2}+y^{2}+z^{2}}\right]\right|_{0} ^{a}
$$

and simplifies to

$$
\vec{E}_{x}=-\frac{\mu_{0} N\left(\frac{d i}{d t}\right)}{4 \pi} \cdot \ln \left[\frac{x-a+\sqrt{(x-a)^{2}+y^{2}+z^{2}}}{x+\sqrt{x^{2}+y^{2}+z^{2}}}\right]_{x}
$$

For the side of the coil at $y=a$, the second element that lies along the $x$ axis, with current moving from $a$ to 0 


$$
\vec{E}_{x}=-\left.\frac{\mu_{0} N\left(\frac{d i}{d t}\right)}{4 \pi} \cdot \ln \left[x-x_{0}+\sqrt{\left(x-x_{0}\right)^{2}+y-a^{2}+z^{2}}\right]\right|_{a} ^{0}
$$

and similarly simplifies to

$$
\vec{E}_{x}=-\frac{\mu_{0} N\left(\frac{d i}{d t}\right)}{4 \pi} \cdot \ln \left[\frac{x+\sqrt{x^{2}+(a-y)^{2}+z^{2}}}{x-a+\sqrt{(x-a)^{2}+(a-y)^{2}+z^{2}}}\right]
$$

For the full expression for the electric field around the coil, the expressions are summed to produce

$$
\vec{E}_{x}=-\frac{\mu_{0} N\left(\frac{d i}{d t}\right)}{4 \pi} \cdot \ln \left[\frac{\left(x-a+\sqrt{(x-a)^{2}+y^{2}+z^{2}}\right) \cdot\left(x+\sqrt{x^{2}+(a-y)^{2}+z^{2}}\right)}{\left(x+\sqrt{x^{2}+y^{2}+z^{2}}\right) \cdot\left(x-a+\sqrt{(x-a)^{2}+(a-y)^{2}+z^{2}}\right)}\right]
$$

Evaluation of the above expression on an $x$ and $y$ plane for a fixed $z$ (height above the coil) will graph the electric field that an axon lying along the $\mathrm{x}$ axis will be exposed to during a current pulse.

As seen in Fig. 6b, a maximum rate of change of the electric field occurs at each corner of the coil and a minimum rate of change occurs in the center of each winding. It becomes useful to have an expression for the spatially varying electric field. This is the source term, or excitation function, for long straight axons. As before, the spatially varying electric field may be evaluated by numerically integrating along the length of the coil
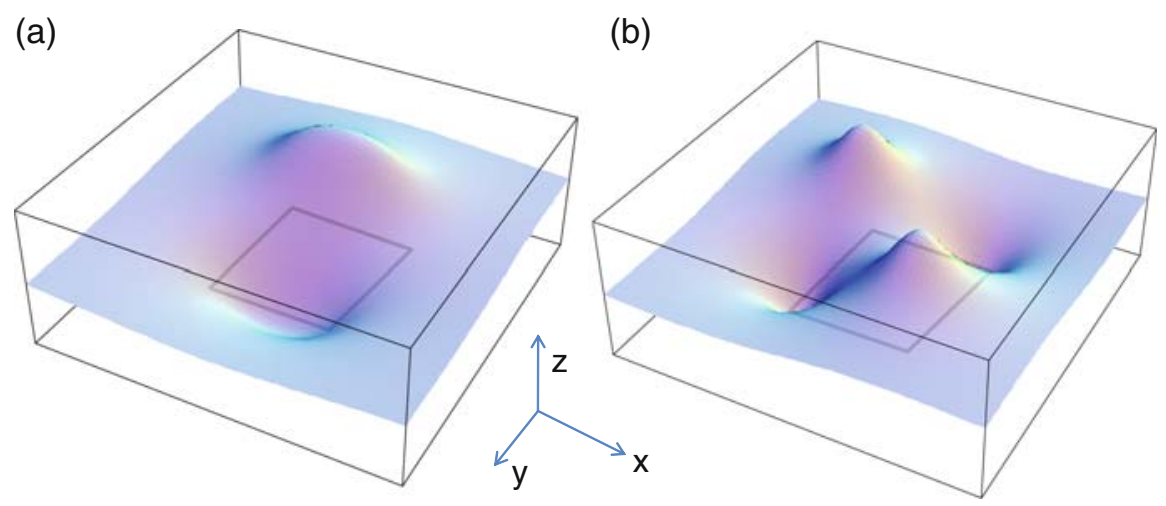

Fig. 6 Fields generated above a unit sized square coil. Square coils are shown in grey. (a) the $\mathrm{z}$ axis shows the relative magnitude of the e field generated above the coil (b) the magnitude of spatial distribution of $\partial \mathrm{E}_{\mathrm{x}} / \partial \mathrm{x}$ maxima and minima generated by square coil. Compare with alternative views in Fig. 3. and Fig. 7 


$$
\begin{gathered}
d\left[\frac{\partial \vec{E}_{x}}{\partial x}\right]=\frac{\mu_{0} N\left(\frac{d i}{d t}\right)}{4 \pi} . \\
\left\{\left[\frac{x-x_{0}}{R^{3}}\right] d l_{x}-\left[\frac{\left(y-y_{0}\right)^{2}-\left(x-x_{0}\right)^{2}}{\rho^{4}}\right] \cdot\left[1+\frac{z-z_{0}}{R}\right] d l_{z}+\left[\frac{\left(x-x_{0}\right)^{2}\left(z-z_{0}\right)}{\rho^{2} R^{3}}\right] d l_{z}\right\}
\end{gathered}
$$

As before, Equation (23) is simplified for the case where the elements of the coil lie in the $\mathrm{z}_{\mathrm{O}}=0$ plane and for square coils with elements lying only along the $x$ axis to

$$
d\left[\frac{\partial \vec{E}_{x}}{\partial x}\right]=-\frac{\mu_{0} N\left(\frac{d i}{d t}\right)}{4 \pi} \cdot \frac{x-x_{o}}{R^{3}} d l_{x}
$$

Alternatively, we can directly evaluate the derivative of the closed form analytical solution for the electric field from Equation (22). The resultant expression after simplification is

$$
\begin{gathered}
\frac{\partial \vec{E}_{x}}{\partial x}=-\frac{\mu_{0} N\left(\frac{d i}{d t}\right)}{4 \pi} . \\
{\left[\frac{1}{\sqrt{(a-x)^{2}+y^{2}+z^{2}}}+\frac{1}{\sqrt{x^{2}+(a-y)^{2}+z^{2}}}-\frac{1}{\sqrt{(a-x)^{2}+(a-y)^{2}+z^{2}}}-\frac{1}{\sqrt{x^{2}+y^{2}+z^{2}}}\right]}
\end{gathered}
$$

The plot of the spatially varying electric field is shown in Fig. $6 \mathrm{~b}$ for the unit coil case $(a=1)$ at a height $(1 / 4 * a)$ above the coil. The figure shows four loci of excitation. The peak electric field and locus of excitation for long straight nerves will occur at the corners of square coil windings as shown in Fig. 3. By setting $y=0$ in Equation (25), the $\delta \mathrm{E}_{\mathrm{x}} / \delta \mathrm{x}$ can be plotted for nerves lying along the $x$ axis. An example at a height of $1 / 4 \mathrm{a}$ is shown in Fig. 7 . The production of a symmetrical anodic and cathodic pulse is consistent with clinical results from the stimulation of myelinated nerves. This demonstrates the possibility of nerve impulse blocking as a function of coil orientation [58].

Fig. 7 Planar slice view of the spatially varying electric field magnitude along the fiber. Fiber is assumed to lie along the $x$-axis

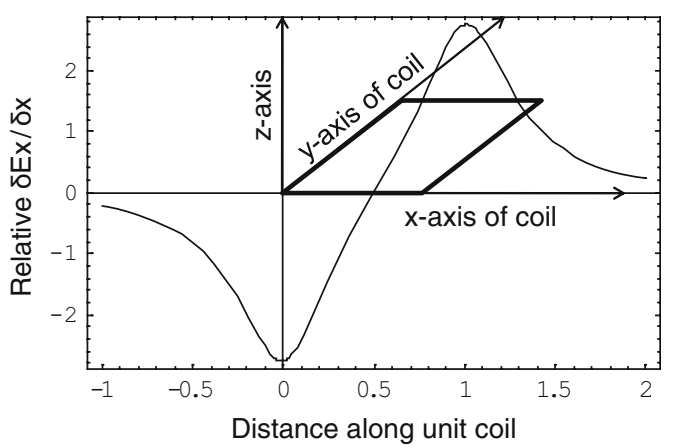


Square coils are employed for three reasons. First, the experimenter can align the nerve tissue with the predicted maximum field. Round coils typically have peaks at the top third and bottom third of the coil, which can be challenging to repeatably locate during experimentation. In square core coils, the peaks align with the corners. Second, a square core coil with no winding elements along the $z$-axis and winding elements parallel to the $x$ and $y$ axis allows an analytical solution to Equations (13) and (23). Third, square coils have been shown in simulation to have approximately $20 \%$ larger effect per unit current than properly aligned round coils [57].

The closed form analytical Equation $(25)\left(\delta \mathrm{E}_{\mathrm{x}} / \delta \mathrm{x}\right)$ provides a design tool for magnetic stimulation. Given the depth of stimulation (z) and threshold of stimulation $\left(\delta \mathrm{E}_{\mathrm{x}} / \delta \mathrm{x}\right)$, requirements for current rise time (di/dt), coil size (a), and number of turns $(\mathrm{N})$ can be directly evaluated. Rapid evaluation of coil and circuit designs and guidance for determining current requirements are possible using this equation.

The stimulus efficiency of different combinations of stimulation depth to coil diameter can be evaluated with the closed form solutions. Setting the $\mathrm{x}$ to 0 (the peak $\delta \mathrm{E}_{\mathrm{x}} / \delta \mathrm{x}$ field), $\mathrm{y}=0$, and making the substitution with the unitless term $\zeta$, which defines the ratio between coil side length and depth of stimulation [59] as

$$
a=\frac{z}{\varsigma}
$$

Equation (25) reduces to

$$
\frac{\partial \vec{E}_{x}}{\partial x}=-\frac{\mu_{0} N\left(\frac{d i}{d t}\right)}{4 \pi} \cdot\left[1-\frac{2}{\sqrt{\frac{1}{\varsigma^{2}}+1}}+\frac{1}{\sqrt{\frac{2}{\varsigma^{2}}+1}}\right]
$$

Similar equations may be derived for double square coils and quad square (or butterfly) coils and show that additional windings at the locus of excitation multiply the effect by either 2 (double square coils) or 4 (quad square coils). In Fig. 8, the results have been plotted. The energy required for magnetic

Fig. 8 Efficiency of magnetic stimulation as a function of distance to site of stimulation and coil geometry. The efficiency of magnetic stimulation falls rapidly as the penetration target depth becomes equal to the coil side length

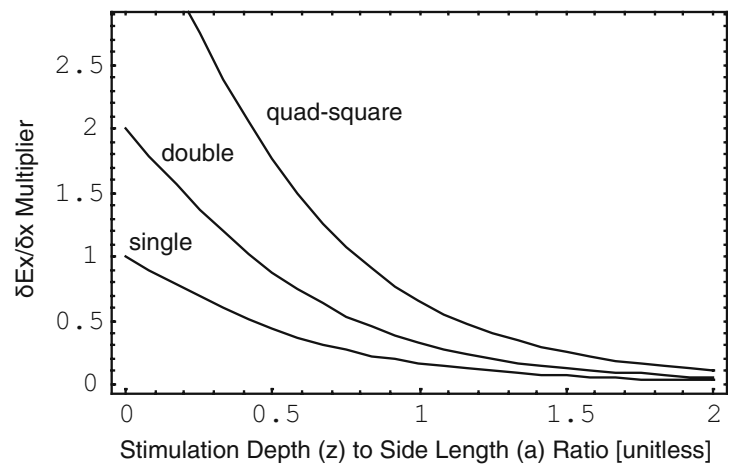


stimulation of nerves increases dramatically as the coil is moved further away from the tissue. The area of effect also increases, leading to less focused area of stimulation. A similar approach is used to analyze quad coil cores with variable intercoil spacing [60].

The value of the load inductance is important for current source design, and in the case of a pulse discharge stimulator system directly affects the stimulus pulse width. An approximation for the inductance of a flat circular coil is [61]

$$
L=\mu_{0} N^{2} r\left[0.48 \ln \left(1+\pi \frac{r}{h}\right)+0.52 \cdot r \sinh \left(\frac{r}{h}\right)\right]
$$

where $\mathrm{h}$ (the height of the coil) is approximated as the thickness of one turn, $\mathrm{r}$ is the coil radius, $\mathrm{N}$ is the number of turns, and $\mu_{\mathrm{o}}$ is permittivity of free space.

Similarly for a flat square coil, inductance is calculated as [61]

$$
L=\mu_{0} N^{2} a\left(\frac{2}{\pi}\right)\left(\ln \left(1+\pi \frac{a}{2 h}\right)+\frac{1}{3.64+4 \frac{h}{a}+4.51\left(\frac{h}{a}\right)^{2}}\right)
$$

where $\mathrm{h}$ (the height of the coil) is approximated as the thickness of one turn, $a$ is the coil side length, $N$ is the number of turns, and $\mu_{\mathrm{o}}$ is permittivity of free space. An alternate calculation method for planar square and round coils is found in [62] and an online calculator is available at http://smirc.stanford.edu/ spiralCalc.html.

The peak $B$ field is often reported as a figure of merit, and so formulas are included for comparative and design estimation. $B$ field formulas allow comparison to clinical experimental systems when B is the reported value. However, a more relevant comparison is the induced electric field or the spatially varying induced electric field.

To calculate the $\mathrm{B}$ field of a ring coil at height of $\mathrm{z}=0$, i.e., the $\mathrm{x}-\mathrm{y}$ plane of the coil

$$
B=\sum_{i} \frac{\sqrt{2} \mu_{o} I}{\pi R} \approx \frac{\sqrt{2} \mu_{o} N I}{\pi R}
$$

where $\mathrm{R}$ is radius of the ring coil in $\mathrm{cm}, \mathrm{N}$ is number of windings, and $\mathrm{I}$ is current in $\mathrm{mA}$. The magnetic induction $\mathrm{B}=\mu \mathrm{H}$ will be calculated in $\mathrm{nT}$. To calculate the $\mathrm{B}$ field at a height above the center of the round coil along the $\mathrm{z}$ axis

$$
B_{z}(z)=\frac{62.83 N I}{R}\left[\frac{1}{\left(1+\left(\frac{z}{R}\right)^{2}\right)^{3 / 2}}\right]
$$

where $\mathrm{z}$ is the distance from the coil center along the coil axis. 
To calculate the B field of a planar square coil at $z=0$, i.e., the $x-y$ plane of the coil

$$
B=\sum_{i} \frac{\mu_{o} I}{a} \approx \frac{\mu_{o} N I}{a}
$$

where $\mathrm{a}$ is the coil side length in $\mathrm{cm}, \mathrm{N}$ is number of windings, and $\mathrm{I}$ is current in $\mathrm{mA}$. The magnetic induction $\mathrm{B}=\mu \mathrm{H}$ will be calculated in $\mathrm{nT}$. To calculate the $\mathrm{B}$ field at a height above the center of the square coil along the $\mathrm{z}$ axis

$$
B_{z}(z)=40 N I \frac{\sqrt{a^{2}+z^{2}}}{2+\left(2 \frac{z}{a}\right)^{2}}
$$

Other coil and nerve arrangements have been presented and have been shown to have advantages over parallel nerve-coil arrangements. Bent nerves are exposed to different extracelluar electric field distributions than straight nerves in the same electric field. If the bend is at the peak electric field, a maximum electric field gradient is induced and the source term is more accurately described as a function of $\mathrm{E}_{\mathrm{x}}$ than of $\delta \mathrm{E}_{\mathrm{x}} / \delta \mathrm{x}$ [18]. The peak electric field and locus of excitation for bent nerves occur midway along the coil windings oriented along the $\mathrm{x}$ axis. A perpendicular coil-nerve arrangement has a very low threshold for stimulation in vitro for relatively long periods of sinusoidal excitation and is also modeled in a simplified manner in [51]. In a perpendicular orientation, however, a comparative application of an electrically induced $\delta \mathrm{E}_{\mathrm{e}} / \delta \mathrm{x}$ can be challenging. Other coil shapes such as the cone [63], crown [64], HESED [65], and Slinky [66] coils used primarily to improve the depth of effect in transcranial magnetic stimulation systems present similar challenges to simple first order modeling. With FEA modeling design verification and careful manufacture, there is no reason alternate coil topologies could not be applied to an in vitro magnetic stimulation system.

\section{Core}

One approach to decreasing the current requirements is to increase the flux in the inductor loop. A core is a very effective way of increasing the magnetic flux. There are several examples in the literature of ferrite and iron cores used for clinical magnetic stimulation [51,67-71] and in vitro experimentation [51, 70, 72-75]. An alternate method of including a core is in [69] where FEA simulation showed a $50 \%$ increase by incorporating a cylindrical plate twice the thickness and $30 \%$ larger than the coil diameter. Cores function essentially as flux concentrators, and electric field is related to flux density as described by Faradays law 


$$
\nabla \times \vec{E}=-\frac{\partial \vec{B}}{\partial t}
$$

The inclusion of a core is analogous to increasing the number of windings and thus the magnetic field capable of generating an electric field for magnetic stimulation

$$
B \propto \mu_{r} \mu_{0} n I
$$

where $\mu_{\mathrm{r}}$ is the relative permeability of the core, $\mathrm{n}$ is the turn density, and $\mathrm{I}$ is current. A straightforward algebraic method to estimate the equivalent permeability, $\mu_{\text {eq }}$, is to employ the reluctance path method (also called the reluctance method, magnetic circuit method, or permeance method). Two excellent resources for further information on this approach are $[48,76]$. The reluctance path method employs an analogy for electric circuits where current (A) is analogous to flux (Wb), electromotive force $(\mathrm{V})$ is analogous to magnetomotive force (A-turns), and resistance $(\Omega)$ is analogous to reluctance $(\Re)$. Figure 9a shows the equivalent magnetic circuit.

(a)

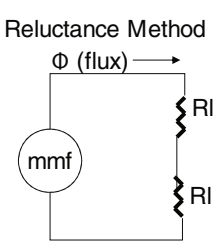

(b)

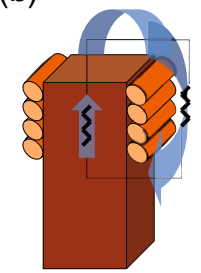

(c)

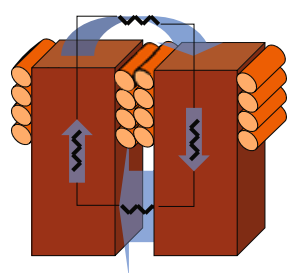

Fig. 9 Reluctance path method for estimating relative permeability. (a) is the reluctance path model abstraction (b) and (c) are examples of square solenoid coils

$$
\Re_{t t l}=\frac{l_{g}}{\mu_{0} A_{c}}+\frac{M P L}{\mu_{0} \mu_{r} A_{c}}
$$

For purposes of estimation, the core becomes a gapped inductor with an equivalent distributed permeability, $\mu_{\mathrm{eq}}$, defined by

$$
\mu_{e q}=\frac{\mu_{r}}{1+\mu_{r} \frac{l_{g}}{M P L}}
$$

where $\mathrm{l}_{\mathrm{g}}$ is distance the magnetic flux travels through (gap length), and MPL is the total length of the flux path. Using the estimated equivalent permeability, the closed form analytical coil equations allow quick estimation of the spatially 
varying electric field component at any point using a variety of core dimensions and core types. For topologies with large air gaps, an improved estimate of inductance is obtained by approximating the fringing flux [77], according to

$$
F=1+\frac{l_{g}}{\sqrt{A_{c}}} \ln \frac{2 \cdot G}{l_{g}}
$$

where $\mathrm{L}$ is the inductance, $\mathrm{F}$ is the fringing flux, $\mathrm{l}_{\mathrm{g}}$ is the summation of the gap lengths, $A_{c}$ is the core area, and $G$ is the height of the winding. The load inductance is then estimated as

$$
L^{\prime}=L F
$$

A further improved method of estimating the fringing flux for more complex geometries is found in [78].

Using cores in stimulation coils allows the reduction of the coil size while maintaining the necessary flux to induce excitation. Two important details come to light using this approach. First, from Equation (25), field focality improves as $\mathrm{z}$ is reduced and the dimensions of the coil are reduced. The resultant scaling allows the interaction of the system with precise structures in the neural tissue under study. Second, the current requirements to generate a $\delta \mathrm{E}_{\mathrm{x}} / \delta \mathrm{x}$ of the correct magnitude fall significantly with the inclusion of a core. Comparable in vitro magnetic stimulation experiments employ di/dt ramps greater than $10 \mathrm{~A} / \mu \mathrm{s}$. By reducing the size of the coil and moving the site of stimulation closer to the coil, the current ramp requirement can be reduced to on the order of $0.16 \mathrm{~A} / \mu$ s (see appendix). Thus, for in vitro experimentation, an improvement in both field focality and significant reduction in energy required is obtained by moving the tissue under study closer to the stimulating coils.

One caveat to using a core as a flux concentrator is that the core must have an operational frequency above the highest frequency component of the driving waveform to reduce power losses due to the core. For single pulse magnetic stimulation systems, iron, steel, and permalloy cores can be used provided they do not saturate. The frequency range for multiple pulse magnetic stimulation requires operation in the $200 \mathrm{kHz}-1 \mathrm{Mhz}$ frequency. Multiple pulse stimulation uses consecutive pulses, as opposed to rTMS, which is a single pulse system at a repetition rate of about $1 \mathrm{~Hz}$. The rapid rise times mandate the use of ferrite cores as the high-frequency operation exceeds most steel core and permalloy core performance specifications. The cores tested in the multiple pulse experiments presented later employ cores from Fair-Rite Products Corporation (www.fairrite.com) of material 77. Performance up to $3 \mathrm{MHz}$ is possible with alternate materials, such as material 61. Custom machining for many shapes is available from several manufacturers such as Elna Magnetics (www.elnamagnetics.com). I cores, E cores, and square $\mathrm{U}$ cores can be used providing they have a square cross section. If the cross section is rectangular, slight modification of the design equations is required. Ferrite cores are essentially a ceramic, and often shatter 
or split when cut. Use diamond bit rotary tool bits and saw blades when cutting $\mathrm{E}$ or $\mathrm{U}$ cores and avoid cutting the wound part of the core.

Further improvements in the resistance loss due to high-frequency effects are possible by using litz wire as opposed to solid core wire or standard stranded wire. Litz wire is a bundle of individually insulated wire woven together to reduce the skin effect and proximity effect losses in conductors. For a closed form design approach to wound components using solid and litz wire, please see [79]. As the current pulse delivered from the magnetic stimulator changes from an undamped and nearly sinusoidal waveform to a damped and less harmonic waveform, core losses become more challenging to estimate. In reality, the processes of energy dissipation in a core under transient non-sinusoidal excitation are considerably more complex. Applying transient currents to ferrite core inductors results in losses from magnetic diffusion time and generation of eddy currents [80-83]. These effects may be severe and challenging to estimate. Since these small core coils are relatively easy to fabricate and few are needed, it is reasonable to estimate their performance, build, test, and tune the inductors.

An impedance analyzer can be an invaluable tool to characterize the fabricated inductors. Since the tissue properties naturally vary so widely from preparation to preparation, the components of a magnetic stimulation systems must perform over a wide range. As long as the stimulation core is properly characterized, analytical evaluation of the data collected is possible. We used a Hewlett Packard 4192A impedance analyzer with a custom interface written in Lab View (code available upon request from the authors) to verify the self-resonant frequency of the coil was above the operating frequency of the circuit, see Fig. 10. The Agilent 4194A and the new 4294A include a built-in equivalent circuit calculator. The impedance analyzers are also helpful for characterizing the pulse discharge capacitors equivalent series inductances and resistances.

Fig. 10 Measurement results of small signal coil impedance. Coil resistance is negligible below $200 \mathrm{~Hz}$, but becomes significant at $2 \mathrm{Mhz}$
Frequency Response of Stimulting Coil

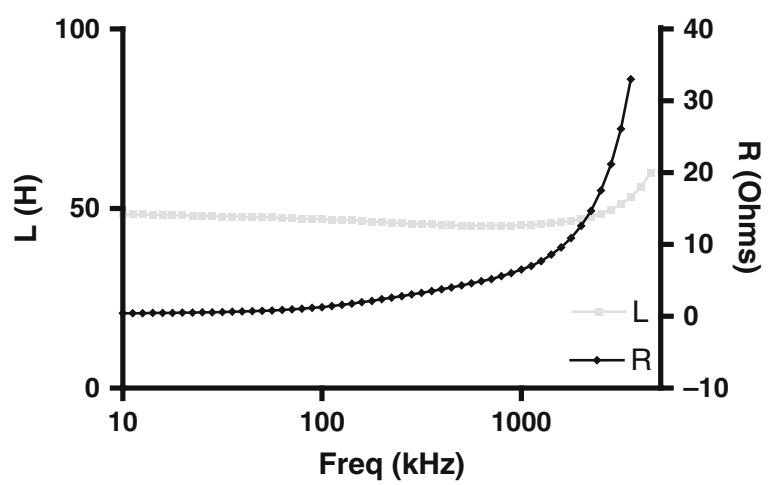


In circuit operation was confirmed by analyzing the Vin/Iout waveform phase shift and shown to have a roll-off frequency above $1 \mathrm{MHz}$. In this manner, the low current and pulsed high-current performance of the cores may be verified. These two techniques were used because the 4192A cannot source high currents and because instrumentation for low frequency, highcurrent inductance measurement is not typically available and would likely overheat the coil as these coils are designed for a pulsed circuit topology, not continuous operation.

\section{Systems for Magnetic Stimulation}

One option for performing magnetic stimulation experiments in the laboratory is to purchase a commercially available system. Commercially available systems can deliver currents and voltages capable of stimulating neuronal tissue centimeters away from the coil, but are expensive because of the necessary interlocks and the inclusion of high-power components. Commercially available systems also are challenging to modify when working with smaller scale systems. Due to their intended use, they may be less configurable for a wide range of pulse shapes and pulse protocols.

Constructing a smaller scale system allows the inexpensive evaluation of new coil excitation circuit topologies. The critical component of a magnetic stimulation system is the power switch used to control current through the load coil. New power devices and pulse capacitors are continually under development [84-86] and supply the need for such devices in industrial applications. Switching strategies and circuit topologies can be tested in a smaller scale system before full power devices become commercially available. For readers interested in the details of full-scale magnetic stimulation system construction, descriptions are available in $[64,87]$. Both of these sources present relatively detailed circuit schematics and application notes. Overviews of magnetic stimulation system design are presented in $[68,88]$.

In the following sections two complete, documented, magnetic stimulation systems suitable for laboratory scale experiments and in vitro magnetic stimulation experiments are presented. The described systems are very dangerous, even though they are reduced in scale. Discharges may reach $100 \mathrm{~J}$. Proper expertise with power electronics and safety protocols are compulsory. The most significant technical standard is IEC-601 Medical Electrical Equipment and there is an excellent approach to safety in laboratory practice in $[87,89]$. Minimally, systems must have appropriate lockouts and the power components must be enclosed. Catastrophic failure of pulse discharge capacitors and the load coil is of particular concern. 


\section{Pulsed System}

Maintaining a current ramp for the length of time required to stimulate neural tissue can confound the design of a current amplifier output stage. Depending on the $\tau_{\mathrm{m}}$ and $\lambda_{\mathrm{m}}$ of the tissue under study, stimulation times range from hundreds of microseconds to a few milliseconds. The common strategy is to use thyristor triggered pulse discharge circuit, as in Fig. 11. The tuned LCR pulse discharge circuit is used to produce a waveform with a damped sinusoidal pulse. The pulse shape is a function of the stimulating coil, the capacitor bank, and the parasitic resistance of the system according to Equation (40).

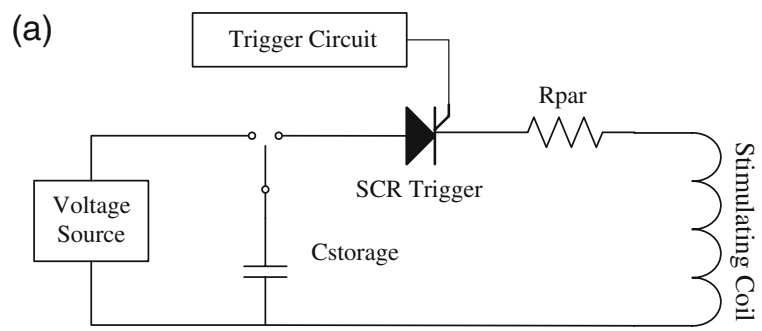

(b)

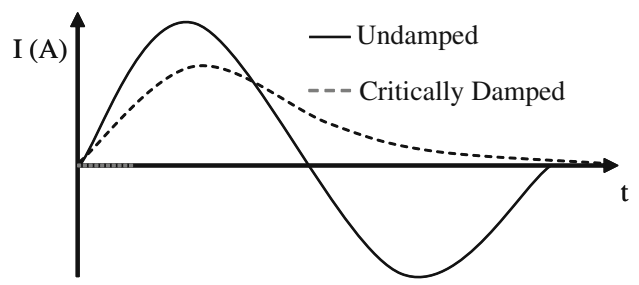

Fig. 11 Typical circuit implementation of a magnetic stimulation circuit. Circuit. (a) A SCR triggered pulse discharge circuit implementation of a time varying current source as in Fig. 1. (b) Resulting current waveform output (b). The system produces different current waveforms and thus different electric fields with varying shape, pulse width and magnitude as a function of the parasitic resistance Rpar in the system, the storage capacitance Cstorage and the inductance of the stimulating coil

$$
I(t)=\frac{V}{\beta L} e^{-\alpha t} \sin \beta t
$$

where

$$
\beta=\sqrt{\frac{1}{L C}-\frac{R^{2}}{4 L^{2}}} \text { and } \alpha=\frac{R}{2 L}
$$

Shorter pulses have been shown to be more energy efficient in pulse discharge circuits [59], while the most energy efficient time point to electrically stimulate 
neural tissue is at the chronaxy of the nerve [90]. Due to losses in the stimulation coil and restrictions on the circuit design window, pulse discharge circuits often operate on much shorter timescales than the chronaxy of the nerve under study.

Undamped systems are called rapid rate stimulators, and up to $40 \%$ of the stimulation energy may be returned to the capacitor bank. While damped systems induce a pair of rectangular electric field pulses, an undamped system produces a set of triphasic pulses [91]. Although the exact mechanism is not known, there is some evidence that stimulus thresholds are lower than the equivalent damped system [88]. This is a conundrum because biphasic electrical stimulation is generally less efficacious than monophasic stimulation.

Recently, a well-documented pulsed discharge system using a novel application of a power device was presented in [64]. The pulsed system we employ in our lab is a modification of this full-scale experimental TMS system. Hopefully, a detailed example will provide insight for users constructing their own systems. A complete system diagram is shown in Fig. 12.

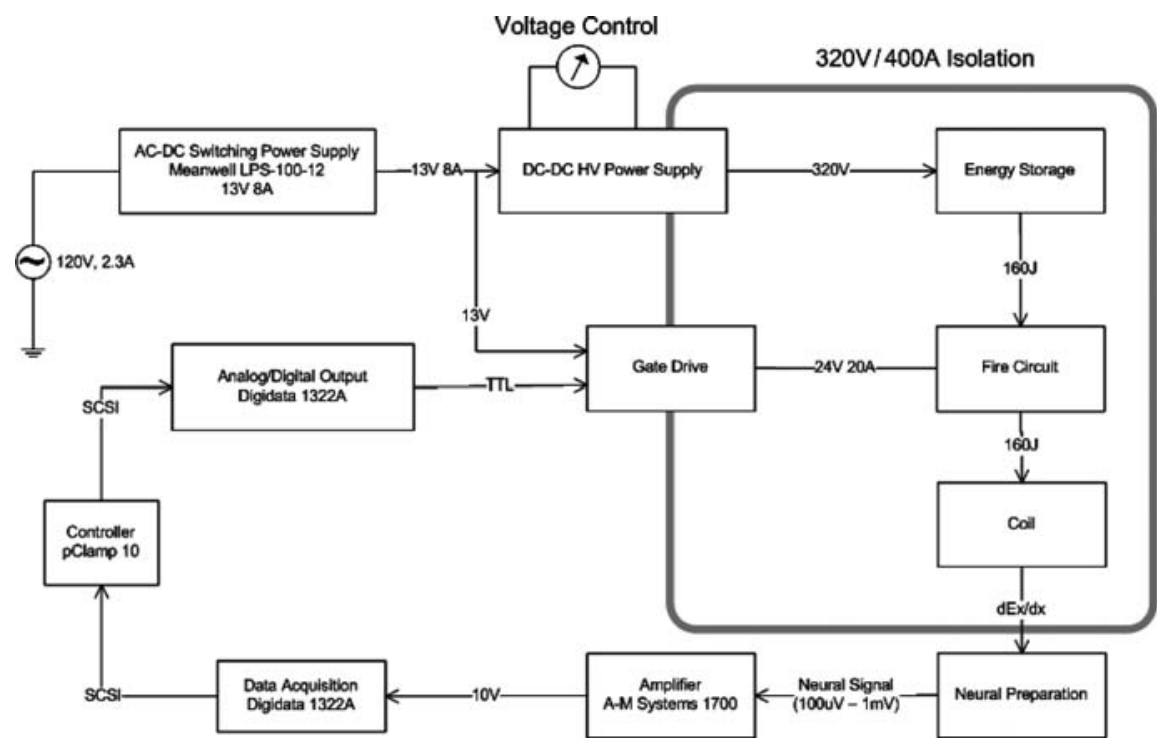

Fig. 12 Block diagram of a pulsed discharge magnetic stimulation and recording system. The grey border shows the electrical isolation area, however, extreme caution must be exercised when using these systems as lethal high voltages are present

A standard switching AC to DC converter (Mean Well LPS-100-12) with isolated outputs provides power to a custom built high-voltage DC power supply. There are several options to provide an adjustable high voltage for capacitor charging, such as a rectified output variac, but flyback transformers [92] have significant advantages for small-scale systems. In contrast to line transformers, flyback transformers utilize relatively small, high-frequency cores so $60 \mathrm{~Hz}$ noise 
near the recording apparatus is mitigated. The LT3750 (Linear Technology, Milpitas, CA) capacitor charger controller is a discontinuous mode flyback transformer intended to charge flash capacitors for consumer applications.

The photoflash capacitors we used leaked significantly until they were held at voltage for several hours. Reforming capacitors is less of a problem than in the past but pulse discharge capacitors can be exposed harsh operating conditions. An auto refresh circuit or regulated charger can also be useful for conditioning capacitors. Linear Technology offers several flyback-based regulated capacitor chargers that employ secondary side sensing, but these do not isolate the primary and secondary parts of the circuit. By adding refresh circuitry, the primary and secondary side can be completely isolated. Auto refresh provides primary-secondary isolation but differs significantly from secondary side sensing in that a runaway condition may occur where charging occurs after the target voltage is reached. To avoid a runaway condition, the capacitor bank leakage should be characterized and the component values for $\mathrm{R}_{\mathrm{t}}$ and $\mathrm{C}_{\mathrm{t}}$ should be set such that

$$
R_{t} C_{t}>\frac{2 I_{P K} L_{\text {Pr imary }}}{I_{\text {Lk }} V_{\text {input }}}
$$

where $\mathrm{I}_{\mathrm{PK}}$ is the leakage current, $\mathrm{L}_{\mathrm{Primary}}$ is the transformer primary inductance, $I_{L K}$ is the capacitor bank leakage current, $V_{\text {input }}$ is the primary side voltage supply, $R_{t}$ is the resistance, and $C_{t}$ is the capacitance shown in Fig. 13. The adjustable flyback-based high-voltage DC power supply can deliver a peak current of 7 A. Voltage is controllable from 20 to $320 \mathrm{~V}$. Several LT3750 devices can be paralleled to provide faster charge rates for higher current charge rates and/or larger capacitances.

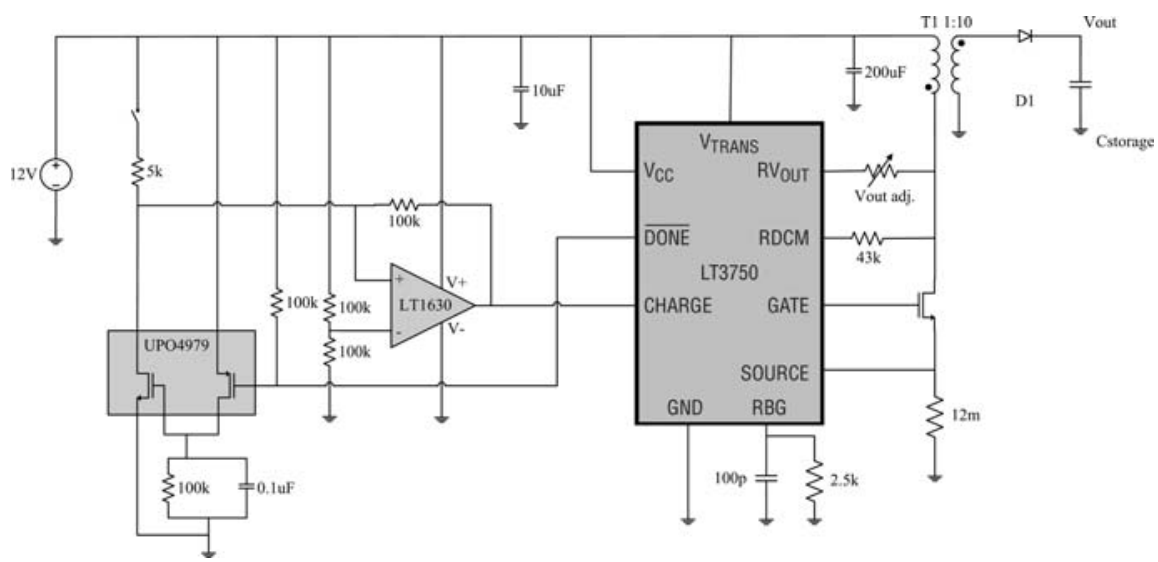

Fig. 13 High voltage power DC power supply. Output voltage is controlled by a refresh circuit. A refresh circuit has an isolation advantage, but runaway conditions can occur. Alternatively a circuit based on the LT3751 can be used to charge the capacitor bank. Flyback power supplies are well suited to laboratory scale magnetic stimulation systems because they provide isolation and be employed in parallel to provide increased current 
Commonly available photoflash capacitors are adequate for energy storage in small-scale magnetic stimulation systems. These typically have an upper voltage limit of $320 \mathrm{~V}$ and are designed for high-current discharge, usually by the designation "photo" on the component markings and specified by the manufacturer for pulse discharge or photoflash applications. It is incredibly important not to exceed the voltage or polarity of photoflash capacitors. Doing either may result in catastrophic failure. Capacitor manufacturers recommended by Linear Technology for the LT3750 include Rubycon, Cornell-Dublier, and NWL. For higher voltage requirements, capacitors can be placed in series or specialized pulse discharge capacitors may be obtained from General Atomics Energy Products, San Diego, CA (www.gaep.com).

Mechanical switches are used to toggle banks of capacitors with values of $100,200,400,800$, and $1600 \mathrm{uF}$. This arrangement allows for variable capacitor storage from 100 to $3100 \mathrm{uF}$. Each capacitor bank includes a discharge circuit to be used before mechanical switching or to safely discharge the storage bank. Mechanical switches are less appropriate for full-scale systems. Full-scale systems that employ banks of capacitors switched by power devices have been presented [93, 94], but these systems can be bulky, complex, and expensive.

A silicon-controlled rectifier cannot stop current flowing once it has been triggered. Only one stimulation pulse width is available for a particular capacitance and load inductance. The controllable pulse-width (cTMS) system employs an insulated gate bipolar transistor (IGBT) to truncate the pulse. Truncating the pulse allows the testing of several time points along a fictive strength-duration curve without a large hardware overhead, which can interrupt experiments. Since the di/dt slope, and ultimately the $\mathrm{E}_{\mathrm{x}}$ and $\delta \mathrm{E}_{\mathrm{x}} / \delta \mathrm{x}$, is set by the voltage stored on the capacitor bank and the load inductance, longer pulse widths can be obtained with a larger capacitance. It is critical to completely discharge the capacitor bank before switching or destructive welding may occur. For both SCRs and IGBTs, it is important not to exceed the maximum pulse voltage, maximum pulse current, or the di/dt and $\mathrm{dv} / \mathrm{dt}$ ratings. While IGBTs can tolerate some current pulses exceeding their operational specs, the maximum collector emitter voltage must never be exceeded. For a comfortable margin of error for flyback conditions, use devices with a greater than required $\mathrm{V}_{\text {ce }}$ rating.

In any high-voltage circuit driving a nearly pure inductive load transient suppression is important. The discrete devices used to shunt inductive spikes, i.e., snubber components, can be mounted directly to the terminals of the IGBT to reduce stray impedance. A diode snubber is available as an integrated component from Cornell-Dublier in the SCM line. WIMA and EPCOS both supply snubber capacitors that mount directly to IGBT modules as well. Use of these devices further reduce terminal impedances. For pulse discharge systems, snubbers and flyback protection are critical. For information on the practical applications of subber circuits, see [95-97].

IGBTs are best operated using gate driver circuits [98]. Gate driver circuits provide sharp transition pulses of the required current to drive the large capacitive gate in an IGBT. IGBT driver circuits can be constructed according to the application notes available on the PowerEx website www.pwrx.com. For lower 
voltages $(<1400 \mathrm{~V})$ and lower current $(<1200 \mathrm{~A})$, PowerEx has made several development kits available, such as the BG2A. A driver board for the CM600 series is also available from Eastern Voltage Research Corporation (http:// www.easternvoltageresearch.com). Voltages exceed $2500 \mathrm{~V}$ in the full-scale cTMS system and a high-voltage optically isolated IGBT driver (AP-1318) is available from Applied Power Systems (Hicksville, NY). All of these IGBT driver systems optically isolate the gate from the input signal. Isolation is important for safety reasons and to protect the computer-controlled TTL outputs. In the scaled system, the high-power components are optically or mechanically isolated from the data acquisition and control unit. Trigger timing is controlled and recorded simultaneously with the output response by a software/hardware data acquisition system. Further information on data acquisition and control for is covered later in the chapter.

As interest in magnetic stimulation rises and new power devices become available, look to sources which cover high-energy pulsed magnetic discharge and circuit design, such as [99, 100]. An alternate approach is presented in [101]. Essentially, the ramp decay rate is controlled by an H-bridge. The transistors in the high-power H-bridge are switched on and off to create a stepped ramp decay.

\subsection{Scaling}

Scaling issues are of particular concern in a pulsed discharge magnetic stimulation system. For a critically damped RLC circuit, $\left(\mathrm{V}_{\mathrm{o}} / \mathrm{L}\right)_{\max }=(\mathrm{di} / \mathrm{dt})_{\max }$ and as a result

$$
\frac{\partial E_{x}}{\partial x_{\max }} \propto \frac{V_{o}}{L}
$$

So the scaling limits for magnetic stimulation depend on inductance and $\delta \mathrm{E}_{\mathrm{x}} / \delta \mathrm{x}$. Inductance scales by $\mathrm{u}_{\mathrm{r}}, \mathrm{N}^{2}, \mathrm{~A}_{\mathrm{c}}^{2}$, while $\delta \mathrm{E}_{\mathrm{x}} / \delta \mathrm{x}$ scales by di/dt, $\mathrm{u}_{\mathrm{r}}, \mathrm{N}, \mathrm{z}$. As shown in Fig. 8, $\mathrm{A}_{\mathrm{c}}$ directly impacts the optimal z, i.e., the ratio of coil diameter to stimulation depth. Setting $V_{\mathrm{o}}$ to a maximum fixed value, as radius increases for a fixed number of turns, $L$ increases and di/dt and thus $\delta \mathrm{E}_{\mathrm{x}} / \delta \mathrm{x}$ falls. Scaling is covered in depth in the appendix and [102]. $\delta \mathrm{E}_{\mathrm{x}} / \delta \mathrm{x}$ and pulse width are both functions of $\mathrm{C}$, $\mathrm{V}_{\mathrm{o}}$, and $\mathrm{L}$ and $\mathrm{R}_{\text {parasitic }}$. A simple spreadsheet can be a valuable way to explore the design space. Analysis should include effects of coil heating. The upper limit to the peak current discharge is often the cross sectional area of the fine wire used in construction of the load coil. Temperature change in a wire is measured by

$$
\Delta T=\rho\left(\frac{I}{A}\right)^{2} \frac{P W}{1 \times 10^{6}} \cdot \frac{c}{\rho_{d e n}}
$$

where $\Delta \mathrm{T}$ is the temperature change in Kelvin, $\mathrm{c}$ is the specific heat $(0.385 \mathrm{~J} / \mathrm{gm} \mathrm{K})$, $\rho_{\text {den }}$ is the wire density (copper is $8.96 \mathrm{~g} /{ }^{\beta}$ ), I is the current in Amps, PW is the pulse time duration, and $\mathrm{A}$ is area in meters. Coil heating and destructive failures are a function of the time the current flows in a wire according to (pp. 4-74 to 4-79 [103]). 


$$
I_{\max }=\frac{A \sqrt{\log \left(\frac{1+T_{m}-T_{a}}{234-\rho_{d e n}}\right)}}{33\left(\frac{P W}{100000}\right)}
$$

where $\mathrm{I}_{\text {max }}$ is current in Amperes, $\mathrm{T}_{\mathrm{m}}$ is the melting point in Celsius $\left(1083^{\circ} \mathrm{C}\right.$ for copper), and $\mathrm{T}_{\mathrm{a}}$ is the ambient temperature in Celsius.

\section{Current Source System}

\subsection{Overview}

Stimulating at the chronaxy of the nerve is the most energy efficient method of stimulation [64, 104]. However, by stimulating near the rheobase of the axon, significant simplification of the electronics is possible. According to Equation (25), the di/dt scales linearly with the $\delta \mathrm{E}_{\mathrm{x}} / \delta \mathrm{x}$ for a fixed coil topology. Reducing the di/dt by half has the effect of reducing the current peak by half. Since $\mathrm{V}=\mathrm{L} \cdot \mathrm{di} / \mathrm{dt}$ is the peak voltage required to obtain that current ramp (ignoring the resistive losses in the circuit for simplification), then this in turn reduces the system voltage requirements by half as well. Power device switching speed varies greatly with the required peak voltage and current rating, which makes a wider array of higher speed semiconductor devices available for different pulsing strategies.

One approach to create longer pulses is to use a sawtooth to generate several concurrent current ramps as in Fig. 14. The asymmetric sawtooth (long rise time, short fall time) also allows a significant reduction in the power dissipated in the inductor as $\mathrm{P}=\mathrm{I}^{2} \mathrm{R}$, provided the waveform has no $\mathrm{DC}$ component. Reducing current and extending the stimulation time should result in significant energy savings, since power is consumed to the square of the current. The

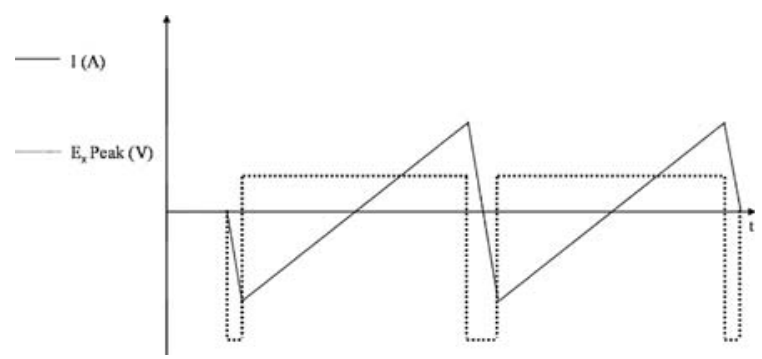

Fig. 14 Sawtooth current waveform and the resultant asymmetric biphasic electric fields produced by coil excitation. The dotted line is the induced E field, the solid line is the current waveform through the inductor 
current supplied to the load is a function of the inductance of the coil when using pulse discharge circuits. In contrast, the current supplied to the load with an asymmetric current source is independent of the load. Decoupling the load from the system performance provides the opportunity to test different inductances without having to redesign the stimulus system or make it overly complex to support a wide experimental range.

Using the sawtooth current driver approach also decouples the induced waveform from the coil electrical properties [38] allowing easier investigation of wave shape variation effects on neural responses. Significant effects of waveform shape have been shown in electrical stimulation modeling [104], confirmed in our lab experimentally and shown clinically for magnetic stimulation in peripheral nerves [105]. Control over waveform shape also enables the evaluation of pulse shape-mediated nerve recruitment [106, 107]. Current driver topologies can deliver much more linear di/dt ramps than a pulse discharge system. The more linear the current ramp is, the more uniform the electric field throughout the course of the pulse, since the induced electric field is a function of di/dt. In clinical functional magnetic stimulation experiments, a constant current ramp has been shown to have lower stimulation thresholds than the commonly used damped sinusoidal waveform [108].

The effects of the polarity of the $\delta \mathrm{E}_{\mathrm{x}} / \delta \mathrm{x}$ waveform may be observed by changing the polarity of the input waveform. A series of pulses also creates an opportunity to test magnetic stimulation effects at longer durations than in the typical pulse discharge circuit. The ability to test the effects of waveform shape on stimulation threshold is a necessary component of a quantitative magnetic stimulation system.

\subsection{Circuit Design}

An analysis of scaling (see Appendix) shows that the current required for smallscale magnetic stimulation (about $\sim 0.16 \mathrm{~A} / \mu \mathrm{s}$ ) is well within the range of a power amplifier topology. It is critical that the transition edge of a sawtooth waveform be as short as possible to prevent hyperpolarizing effects on the neural membrane, or worse, inactivating the $\mathrm{Na}^{+}$ion channels, thereby increasing the energy requirements for stimulation. A grounded load V-I converter, such as an improved Howland VCCS (Fig. 15) [109], is typically used in this application. For a typical Howland current source, the output impedance falls as the signal frequency increases. According to Equation (46), the output impedance appears capacitive as the effects of finite open loop gain come into play [110]

$$
C_{e q}=\frac{R_{1}+R_{2}}{2 \pi f_{o} R_{3} R_{4}}
$$

where $\mathrm{f}_{\mathrm{o}}$ is the gain bandwidth product of the amplifier, and R1, R2, R3, and $\mathrm{R} 4$ are resistances as in Fig. 15. When driven with a sharp transition pulse past 
Fig. 15 Improved howland current source (inverting). R3 functions as the sense resistor. The coil is not part of the feedback loop

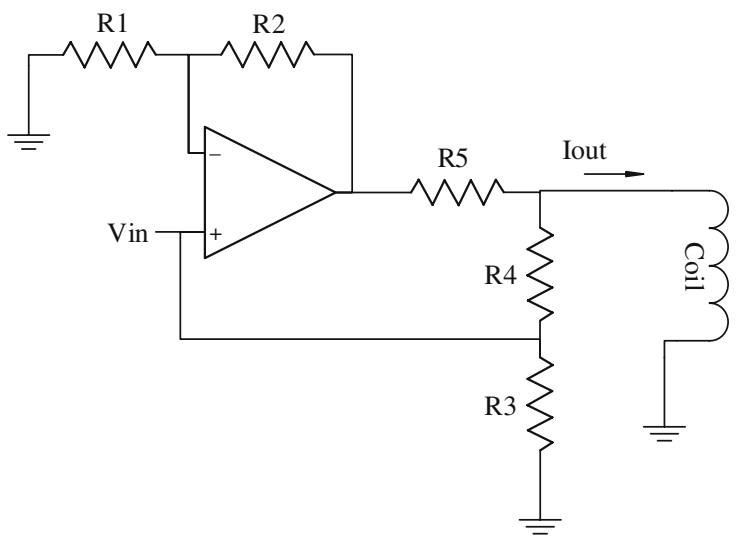

the unity gain bandwidth frequency, the lowered output impedance of the amplifier and the nearly pure inductance of a magnetic stimulator coil form an LC tank. The driver circuit becomes unstable as evidenced by high-frequency oscillations at the output (see Fig. 16). The drop in amplifier output impedance is often further degraded by the desire of the designer to employ low-value resistances in the feedback path to improve frequency response. Small feedback resistance values reduce the output impedance and degrade accuracy due to component tolerance variation.

The more appropriate approach is to include the reactive load inside the feedback loop. The floating load current source topology, Fig. 17, is particularly

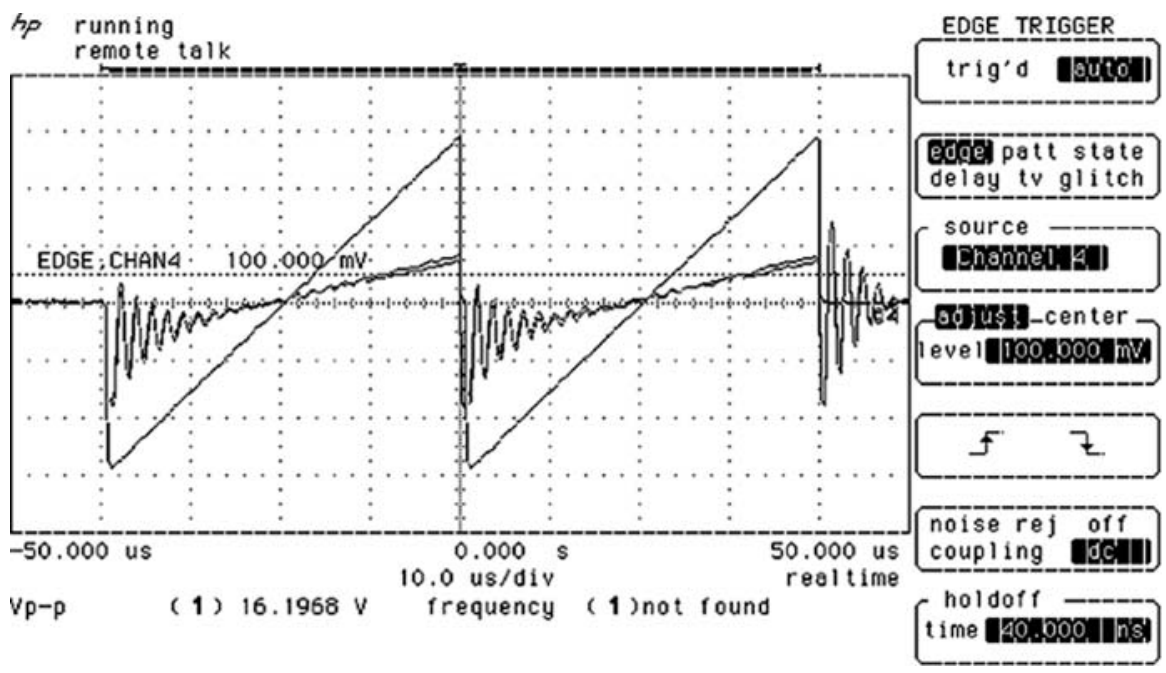

Fig. 16 Ringing at output of inductively loaded current source. Typical results obtained by loading a current source with a nearly pure inductive load and a sawtooth input waveform. The effective output capacitance can be estimated by measuring the frequency of the ringing waveform when the inductor is excited with a step input 


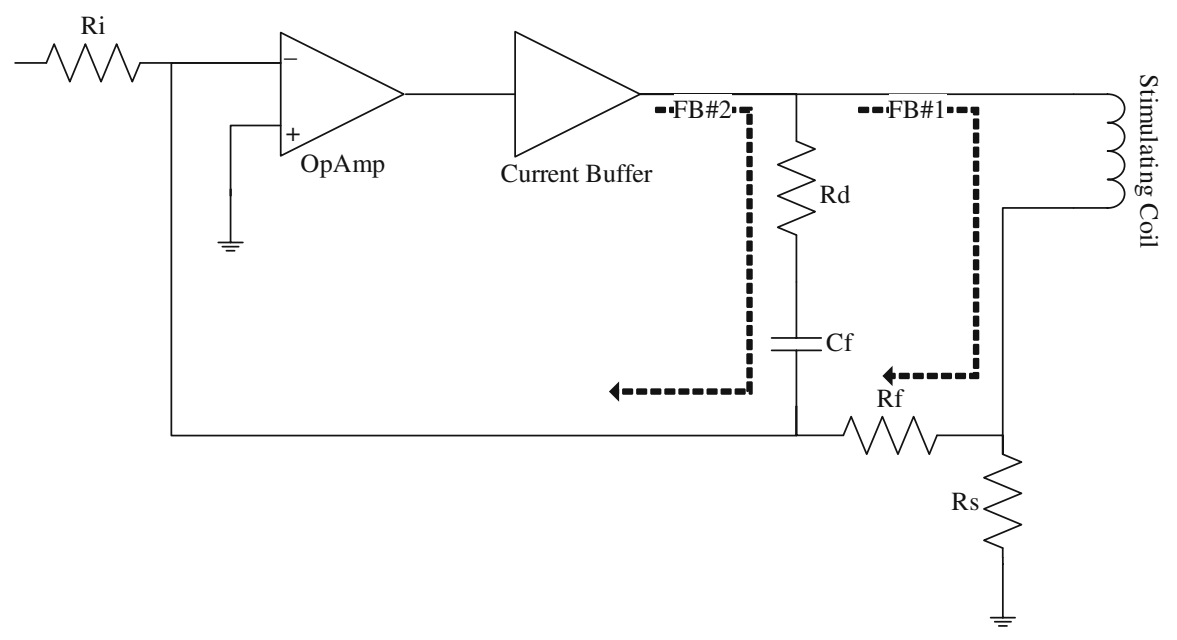

Fig. 17 Floating load current source topology. The operational amplifier and the current buffer function as a composite amplifier. By including the load coil in a feedback loop current waveforms can be supplied to the load while maintaining stability. A second feedback path provides additional high frequency stability and improved step transition response

appropriate for magnetic stimulation as the coil is always isolated from the preparation (and the patient in clinical applications). Both terminals of the inductor are available and it may be placed into the feedback loop and compensated. The floating load current source is insensitive to component tolerance mismatch that plagues the improved Howland current source. In Fig. 17, Rs functions as a sense resistor, thus the current across the load is defined as

$$
I_{\text {load }}=-\frac{V_{\text {in }}}{R_{s}} \frac{R_{f}}{R_{\text {in }}}
$$

\subsection{Rate of Closure Stability Analysis}

The method used to analyze this circuit uses rate of closure feedback loop analysis [111]. The open loop gain of the amplifier is plotted. The feedback factor, $\beta=V_{f b} /$ $\mathrm{V}_{\text {out }}$, of each independent feedback path is calculated and then the inverse $(1 / \beta)$ is plotted. The closed loop gain follows the lowest feedback path, as in Fig. 18. The difference between the closed loop gain and the open loop gain is the circuit's loop gain and graphically equivalent to the area between the feedback plot and the open loop gain. For the case of the floating load current source, the DC loop response is calculated when the inductor is shorted as

$$
V_{f b}=V_{o u t} \frac{R i}{R f+R i} \cdot \frac{R s(R f+R i)}{R l R s+R f(R l+R s)+R i(R l+R s)}
$$




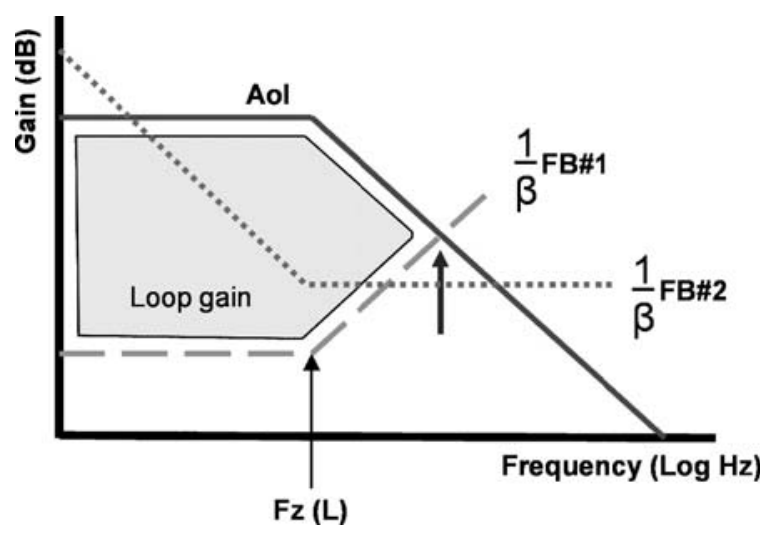

Fig. 18 Beta feedback Analysis for multiple feedback paths. The dashed line is the feedback path formed by the inductor, $\mathrm{Rf}$ and $\mathrm{Rs}$. Fz (L) denotes the zero created by including the active load in the feedback loop. The dotted line is the feedback path through the Rd and Cf high frequency shunt. The solid line represents the open loop gain of the amplifier and the buffer stage. The arrow denotes a loop closure rate of $40 \mathrm{~dB}$ when the second feedback path is not present indicating an instability condition. By including a second feedback path with a zero the closed loop response (i.e. following the lowest line across the bode plot) splits the $40 \mathrm{~dB}$ loop closure rate into 2, $20 \mathrm{~dB}$ loop closures

The first feedback loop (FB\#1 in Fig. 18) is through the inductor and the zero in the feedback path is defined by the inductance and parasitic resistance of the load as

$$
f_{z}(L)=\frac{R s-R l}{2 \pi f L_{\text {load }}}
$$

If a second feedback path is not in place, the feedback loop and the amplifier's open loop gain plot intersect with a closure rate of $40 \mathrm{~dB}$. A rate of closure of $40 \mathrm{~dB}$ corresponds to a phase shift of $180^{\circ}$ and oscillatory behavior. To compensate, a second feedback path with a feedback path zero (capacitor) is added to the circuit (FB\#2 in Fig. 18). The value of the capacitor is set below the feedback path of the load feedback path and the resistance is set to provide a rate of loop closure of $20 \mathrm{~dB}$ and also at least $20 \mathrm{~dB}$ greater than the DC gain $[110,112]$. The second feedback loop zero is defined by the feedback capacitor and the feedback resistance as

$$
f_{z}(C)=\frac{1}{2 \pi R_{d} C_{f}}
$$

Rate of closure is a powerful technique, but for a complete analysis it is important to check the open loop phase shift throughout the gain bandwidth. This may be estimated using hand plots or spreadsheets [113], but the use of Tian's method [114] for feedback loop analysis is more accurate and handily implemented in SPICE. 
SwitcherCAD from Linear Technology bears mention [115]. SwitcherCAD is a free SPICE program available from Linear Technology. It is well supported by an active internet community (LT SPICE at yahoo groups), which includes a wide variety of uploaded test jigs and component SPICE models. For example, Tian's and Middlebrook's methods for feedback loop analysis are implemented directly in examples. It uses a compact description for component parasitic modeling that greatly improves both accuracy and speed, and improves convergence for simulation of pulsed systems.

\subsection{Output Stage Design Details}

There are a few implementation caveats to the floating load current source topology approach. Inductance of the sense resistor must be minimized to prevent peaking and oscillation. The value of $R_{\mathrm{s}}$ impacts the loop gain and larger values improve both the power bandwidth and the settling time according to Equation (49). In this topology, the entire current flows through the current sense resistor and larger values will increase the voltage drive requirement. Unfortunately, monolithic operational amplifiers that have the necessary gain bandwidth and drive current in the ampere range are not available. To meet this need, we built a composite amplifier using a commercially available current feedback amplifier (LT1468) and implemented a discrete power output stage. Current feedback amplifiers are ideal for this application as they provide moderate gains and a high unity gain bandwidth frequency with excellent drive capabilities.

The power output stage bipolar junction transistor (BJT) power devices were selected because of their low output resistance, reduced drive requirements, and immunity to inductive kickback spikes, which can destroy field effect metal oxide semiconductor transistors. However, it is generally difficult to find radio frequency (RF) power PNP devices. The lack of high-speed PNP devices can be resolved by using composite devices [116]. Careful design is important because composite devices may develop local oscillations and have bandwidth limitations.

Composite devices (Darlington or composite PNP) are required because the gain of power RF BJT devices is generally low. RF devices are used because the current booster stage must be significantly faster than the driving operational amplifier. Figure 18 shows the speed requirement of the current booster stage. If the current booster stage has a $3 \mathrm{db}$ roll off below the unity gain bandwidth of the amplifier, it will introduce an additional pole and invite oscillation. If the current feedback stage cannot respond to the output control signal from the amplifier, then the output will oscillate while the current booster continually tries to catch up to the feedback signal measured at the sense resistor. To prevent the addition of poles in the feedback loop, the midband gain of the power stage must extend past the unity gain frequency of the amplifier [117, 118].

Placing a reactive load inside the feedback loop requires particular attention when switching currents. Switched currents result in sharp flyback 
pulses from the inductive load. Flyback voltage pulses can damage components and create intermittent failures. The most effective way to dissipate flyback voltage pulses is to include discrete ultrafast recovery flyback diodes (Fig. 19, clamping diodes shown on the right). With proper selection of components the parasitic capacitance added to the output is negligible. Additional protection from flyback pulses is achieved by using unidirectional zener diodes on the power supply rails [119].

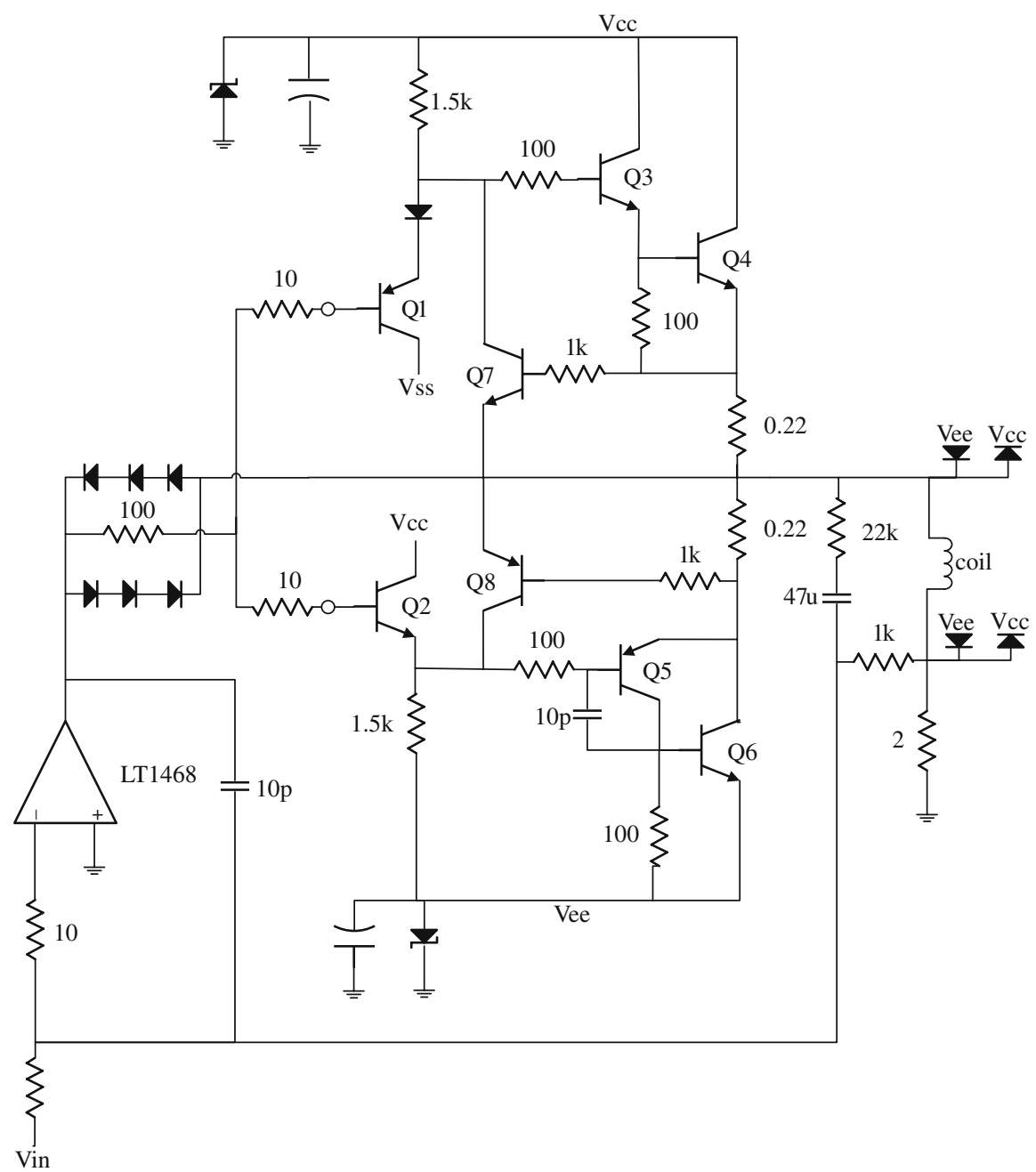

Fig. 19 Reactive load driver circuit. Example of a complete linear current source circuit with the reactive load inside the feedback loop. Circuit is capable of delivering 4App. Transistors are Advanced Semiconductor Q1,Q8 $=2$ N2907 Q2,Q7 $=2$ N2222 Q3 $=2$ N3866 Q5 $=2$ N5160 Q4, $\mathrm{Q} 6=2 \mathrm{~N} 3632$, input diodes are $1 \mathrm{~N} 4148$, clamping diodes are BAV99, zener deiodes are 1.5KE16A, electrolytic caps are low ESR, 2200uF. Vcc and Vee are $+16 \mathrm{~V}$ and $-16 \mathrm{~V}$ respectively 


\subsubsection{Scaling}

The advantage to using a controlled current source rather than a pulse discharge circuit is that the waveform delivered is nearly independent of the load coil. Different coil topologies may be directly compared, and waveforms reversed in polarity and changed in shape and duration during experimentation.

\subsection{Circuit Testing}

In the following, we present several methods of testing and evaluation of the sawtooth current generator. Hopefully, examples of testing will help users characterize and debug their systems quickly.

\subsubsection{Verification of the Current Waveforms}

The entire system was tested with a range of inductances from 5 to $100 \mu \mathrm{H}$, with and without cores, to verify whether system was stable. The test inductors ranged from low parasitic resistances of $10 \mathrm{~s}$ of milliohms to $1 \mathrm{Ohm}$. Figures 20,21 , and 22 show typical simulation and measurement results from a $20 \mu \mathrm{H}$ coil. It is important to use precise probing techniques as the inductance of the probe placed on the output terminal to the coil can influence the measured waveform for this circuit topology.

Fig. 20 Increasing current waveform response of reactive load driver circuit. Measured responses of ramp pulse swept in increasing input voltage for the reactive load driver circuit. (a) input voltage waveforms

(b) measured voltage across the $2 \mathrm{ohm}$ sense resistor, similar to line $\mathrm{C} 2$ in Fig. 21
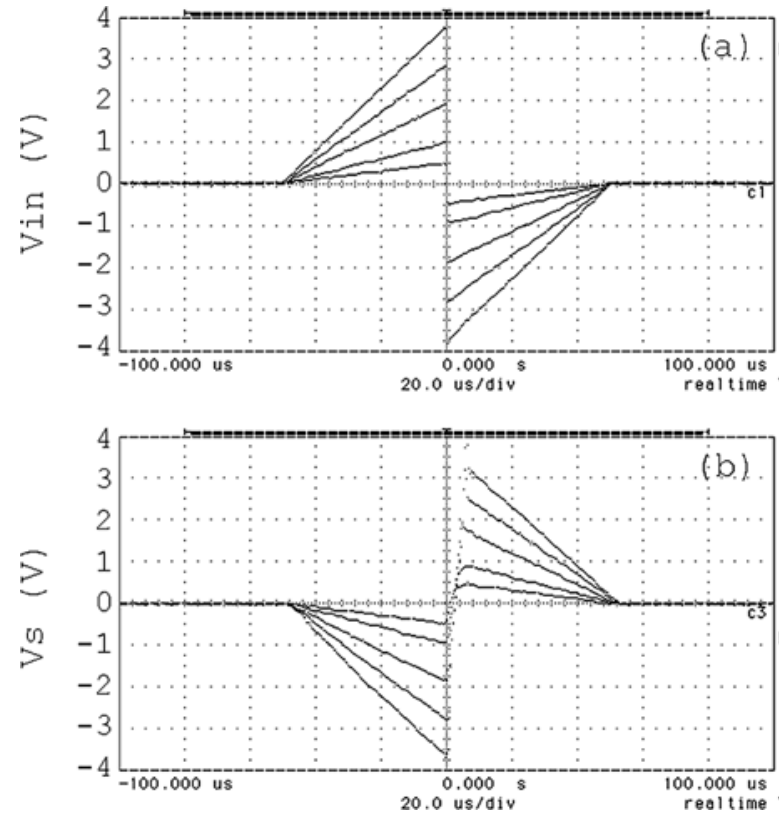
(a)

(b)
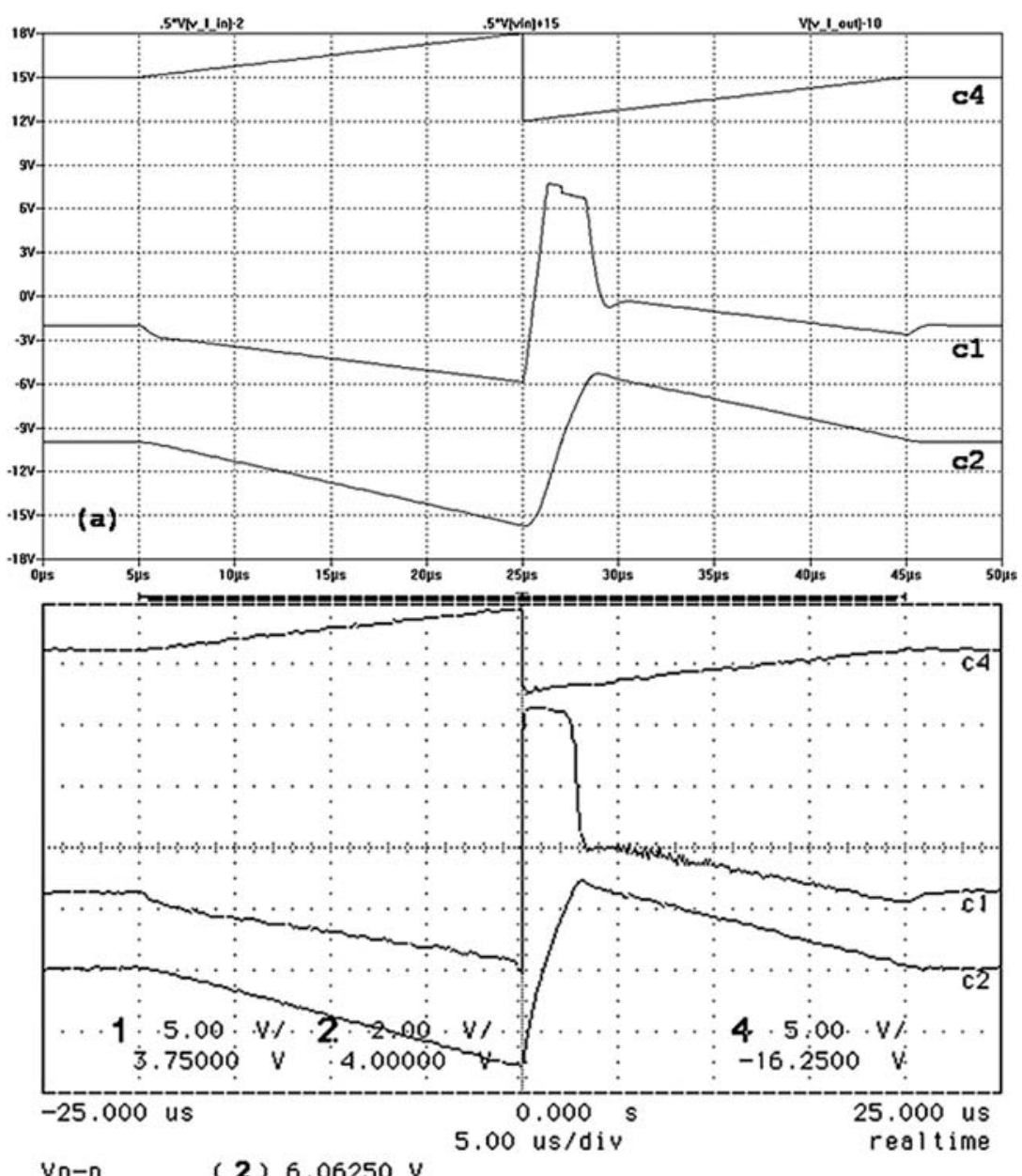

Fig. 21 Simulated and measured responses of a single ramp pulse for the reactive load driver circuit. (a) Waveforms from LT Spice simulation (b) measured response. C4 is the input waveform, $\mathrm{C} 1$ is the voltage at the load, $\mathrm{C} 2$ is the voltage across the sense resistor

Fig. 22 Frequency performance of reactive load driver circuit. Sine wave sweep of increasing frequency at half power measured at the sense resistor. The $3 \mathrm{db}$ roll off was measured at $129 \mathrm{kHz}$, indicated by bold arrow

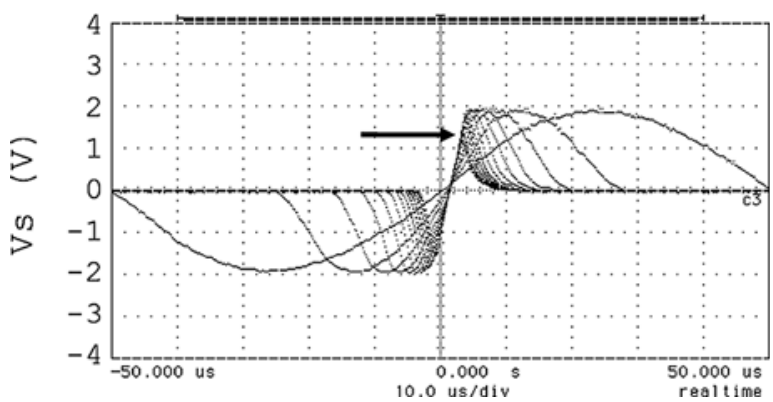




\subsubsection{Verification of the Electric Field}

To verify that the electric field was generated by the time-varying current pulse as predicted, a pickup coil was placed over the coil as the circuit generated single current sawtooth waveforms. The signal from the pickup coil was amplified using an INA111 instrumentation opamp (Texas Instruments, TX) with a gain of 10X. The TI INA111 has a very high-frequency response and an adjustable gain. Users should carefully consider the peak amplitude of the induced potential and include an input protection network for testing their coils if necessary. The electric field produced from a triangular current ramp should be a square wave as shown in Fig. 23. While [120-122] discuss probe construction for measuring electric fields above and inside solenoid inductors, probe construction, accurate measurements, and proper alignment on the submillimeter scale proved unreasonable.

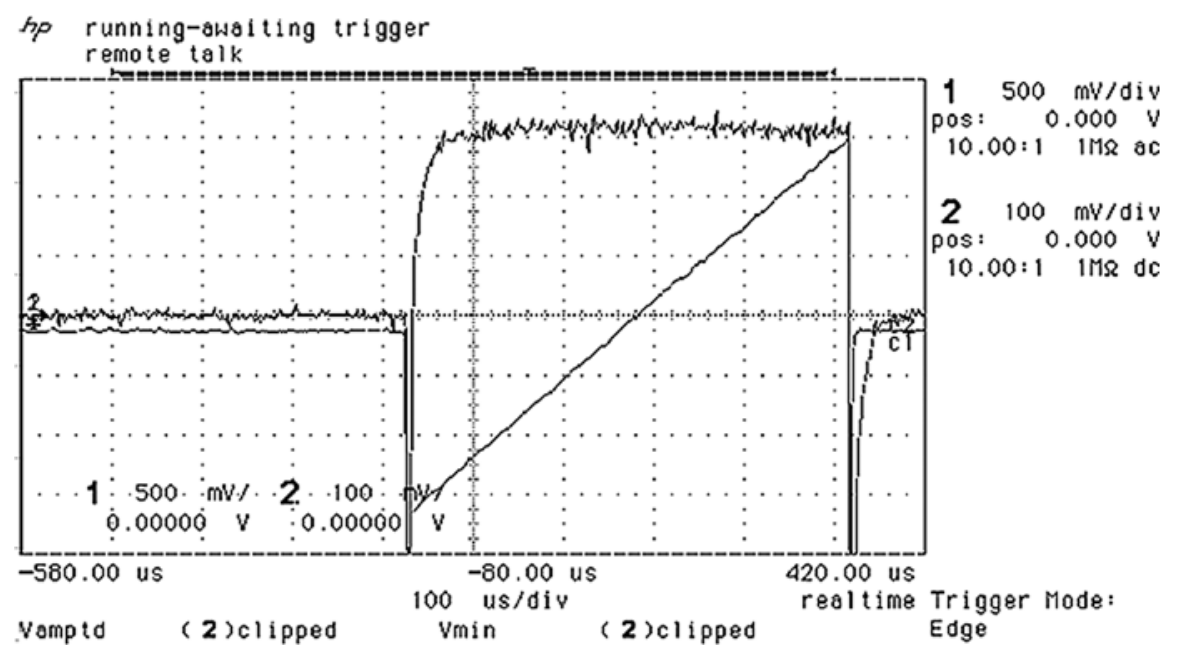

Fig. 23 Measurement of electric filed generated in situ. Ramp output waveform and measured response from pickup coil area shown

\section{Neural Preparations}

Required excitation threshold is determined from the physiological parameters of the neural model under study. As previously discussed, the spatial rate of change of the electric field along the length of the axon $\left(\partial \mathrm{E}_{\mathrm{x}} / \partial \mathrm{x}\right)$ has been shown to predict extracellular neural electrical stimulation (see review in [30]). Clearly, the spatial rate of change of the electric field along the length of the axon is one experimental variable. However, the space and length constants of the tissue under study are also 
experimental variables. Little study has been completed to investigate the effect of waveform shape and pulse-width variation on preparations with varying time and length constants. Both the length and the time constants are functions of passive membrane properties $r_{m}, r_{i}, c_{m}$ (Equation 51), which depend on membrane surface area as well as its intrinsic resistive and capacitive characteristics such as density of ion channels and membrane thickness and determine both the variation of excitability of the cell and the propagation speed of the action potential.

$$
\lambda_{m}=\sqrt{\frac{r_{m}}{r_{i}}} \text { and } \tau_{m}=c_{m} r_{m}
$$

In Equation (52) $C_{m}, R_{m}, R_{i}$ are the size-independent-specific membrane properties in $\left(\mathrm{F} \cdot \mathrm{cm}^{-2}\right),\left(\mathrm{k} \Omega \cdot \mathrm{cm}^{2}\right)$, and $\left(\Omega \cdot \mathrm{cm}^{-2}\right)$, respectively; a $(\mathrm{cm})$ is the fiber radius:

$$
c_{m}=C_{m} 2 \pi a \quad[\mathrm{~F} / \mathrm{cm}] \quad r_{m}=\frac{R_{m}}{2 \pi a} \quad[\mathrm{k} \Omega \cdot \mathrm{cm}] \quad r_{i}=R_{i} / \pi a^{2} \quad[\Omega / \mathrm{cm}]
$$

In the absence of myelination, diameter variations control the efficacy of neural signaling by determining the length constant, which is proportional to the square root of the radius of the process [123]. Larger processes have both lower thresholds for extracellular stimulation and faster propagation velocities, resulting in longer length constants. In the ideal case where the cell can be modeled by a lumped $\mathrm{r}_{\mathrm{m}} \mathrm{c}_{\mathrm{m}}$ model, the time constant $\tau_{\mathrm{m}}$ is independent of axon diameter, since $c_{m}$ and $r_{m}$ are reciprocally dependent on the size of the cell. In this idealized case, $\tau_{\mathrm{m}}$ is a measure of the excitability of the cell that depends on its intrinsic properties rather than size. Thus, both different cell types (motor neuron versus sensory neuron) and different areas of the cell (axon hillock versus soma) may have different time constants, and thus different excitabilities, with shorter time constants corresponding to faster signaling.

Experimentally, $\lambda_{\mathrm{m}}$ and $\tau_{\mathrm{m}}$ can be elucidated from published or directly measured passive membrane properties as well as the strength-duration curve. Membrane capacitance depends on the intrinsic properties of the phospholipid bilayer, with the specific capacitance per unit area of all biological membranes, $C_{m}$, equaling approximately $1 \mu \mathrm{F} / \mathrm{cm}^{2}$. A useful tool for measuring membrane properties in the lab is Neurofit [124], a MATLAB-based software package that allows the extraction of active as well as passive properties of the membrane from voltage-clamp experiments.

Experimental derivation of a strength-duration curve using a point source electrode is the first step to magnetic stimulation experiments, because it allows the extraction of a series of stimulus magnitudes and durations that are sufficient to stimulate tissue at a specific distance between fiber and current source. Additionally, qualitative analysis of the curve shape can tell important things about passive properties of the neural preparation. At rheobase, the membrane 
is essentially voltage-clamped at just below threshold voltage, and the stimulus current is equal to ionic current. According to Equation (53), $\mathrm{I}_{\mathrm{rh}}$ is dependent on and will increase with the size of the cell:

$$
V_{t h}=\lim _{t \rightarrow \infty}\left[I_{t h} r_{m}\left(1-e^{-t / \tau_{m}}\right)\right]=I_{r h} r_{m}
$$

Thus, rheobases of cells with a larger diameter will be shifted up on the strength-duration curve.

The chronaxy, or the minimum pulse duration needed to reach $\mathrm{V}_{\text {th }}$ when pulse strength is twice the rheobase, estimates a pulse length with a reasonably low stimulus. With some limitation chronaxy provides a relationship between an experimentally derived strength-duration curve and the time constant:

$$
t_{\text {chronaxy }}=\tau_{m} \ln 2
$$

Thus, while rheobase is dependent on the surface area of the cell, the chronaxy depends on the specific membrane resistance, and thus on density of ion channels, rather than size of the cell. However, Equation (53a) is derived from the isopotential lumped $\mathrm{r}_{\mathrm{m}} \mathrm{c}_{\mathrm{m}}$ model, where the stimulus is expressed in terms of transmembrane current. In axons, and cells with non-spherical morphologies, as well as experiments where the stimulus is a change in extracellular potential gradient, this relationship is not as clear, and chronaxy does exhibit some dependence on fiber shape, size, as well as distance of point current source from fiber [125]. However, this variation is much less significant than the variations we see in rheobase and the space constant [125]. Generally, the chronaxy estimates a pulse length with a reasonably low stimulus that is sufficient to depolarize the cell. In general, faster-signaling fibers exhibit shorter chronaxy values.

In the case of extracellular stimulation of an axon trunk, this variation in passive properties leads to the recruitment order where larger diameter axons are recruited at lower stimulus magnitudes than smaller diameter ones.

While this recruitment order may be convenient in terms of experimentation, since it allows the excitation of a small subgroup of large axons while stimulating an entire nerve trunk, it also makes it difficult to selectively stimulate smaller axons without stimulating larger ones. It is possible to suppress the extracellular voltage gradient for fibers within a specific diameter range, by using arrays of multiple electrodes [126], however, for magnetic stimulation this would require a number of closely spaced coils.

Having neural preparations with a variety of fiber diameters, as well as a spatially defined distribution of axon diameters, facilitates recruitment studies by enabling the selection of axon fiber by distance from stimulus source as 


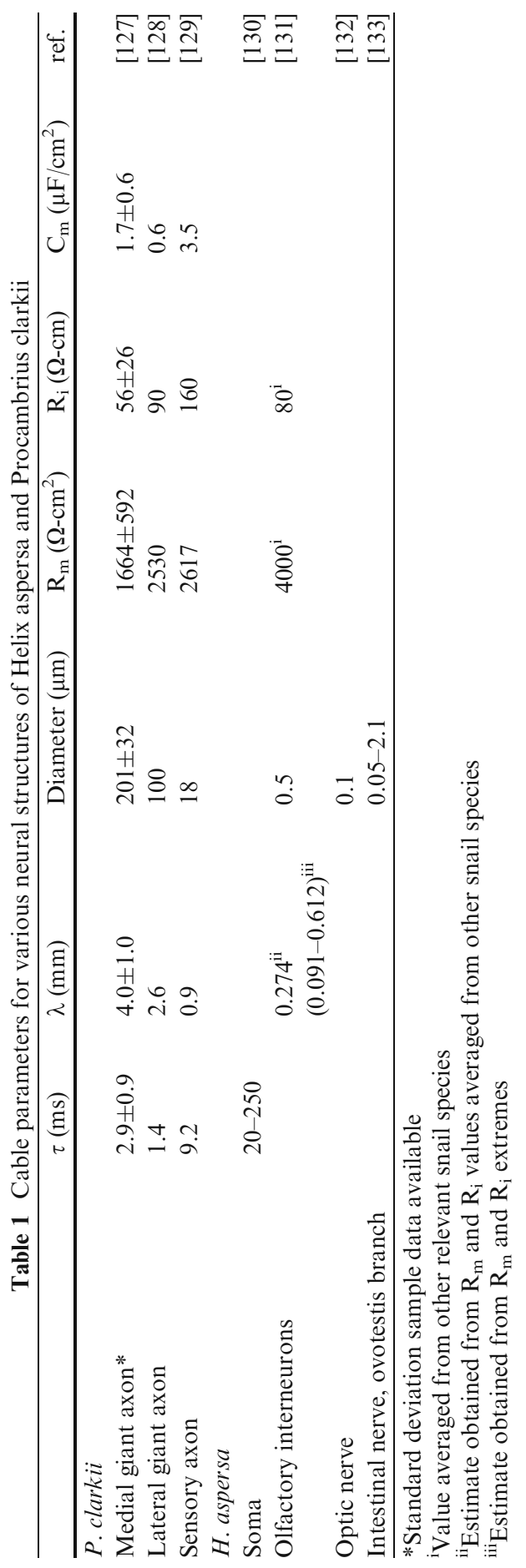


well as by fiber diameter. The medial and lateral giant axons in the crayfish abdominal nerve cord are an excellent example. Table 1 displays some useful cable parameters for two neural preparations discussed in the following section.

\section{Selection}

In selecting a neural preparation, several factors should be considered. Specific structures must be repeatably and reliably located to allow testing of similar structures from experiment to experiment. The preparation should be asymmetric - offering both large and small axons for study of recruitment and different stimulus thresholds. Spatially defined nerve morphologies allow the experimenter to explicitly determine the distance from source to fiber. Stability under a wide temperature range allows alteration of the temperature-dependant length constant. Preparations that are $\mathrm{pH}$ insensitive permit short-duration charge-imbalanced stimulation, which greatly simplifies modeling of electric fields used for control experiments. Invertebrate preparations meet these criteria and have proved to be an invaluable neurobiological model [134]. In addition, axons of these organisms are unmyelinated, which further simplifies correlation between modeling and experimental data. Unmyelinated nerves may also serve as a model of human pain afferents in pulsed electromagnetic field pain therapy [1] and for studies on unmyelinated axons in the human brain and organs [135].

The use of a stimulating coil with cores allows the use of physically smaller neurobiological preparations. Here we use two neural preparations to evaluate the effects of magnetic stimulation on unmyelinated nerves and demonstrate magnetic stimulation with a ferrite core stimulation coil.

The abdominal nerve cord from $P$. clarkii makes an excellent neurobiological preparation because it is extensively studied. The nerve cord has several structures that are easy to locate and allow the orientation of known morphological structures. The lateral and medial giant axons are easily located (Fig. 24). In addition, crayfish have several sensory non-spiking afferents that may permit the study of magnetic stimulation effects on non-spiking neurons.

The circumesophageal ring from $H$. aspersa (Fig. 25) is another useful model. $H$. aspersa makes an excellent model because the circumesophageal ring contains individual cells and nerves that can be located repeatably [136]. Specifically, the pedal and optic nerves serve as excellent material for relatively long, uniform nerves. Moreover, the snail eye stalk containing both the olfactory and the optic nerve is susceptible to tactile stimulation [137]. The length and time constants of $H$. aspersa and $P$. clarkii are also greatly different, permitting a range of experimental conditions to test the validity of Equations (6) and (8).

Both of these neurobiological preparations are thoroughly documented as part of a laboratory curriculum [138, 139], and specimens are inexpensively obtained from biological supply houses. The space constant may be varied by 
Fig. 24 Transverse section of the crayfish abdominal nerve cord. Lateral Giant neuron (LG) and Medial Giant neuron (MG) are clearly seen near the top (dorsal side) of the figure. Black bar is approximately $100 \mu$ M. Reprinted with permission from [158]
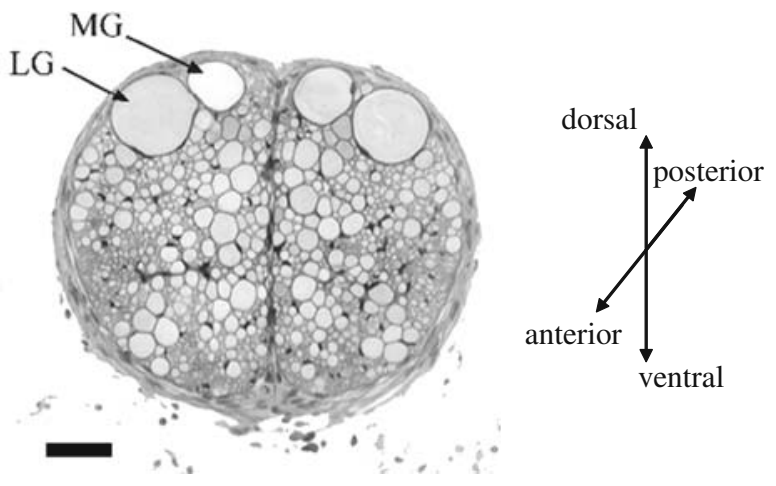

a.

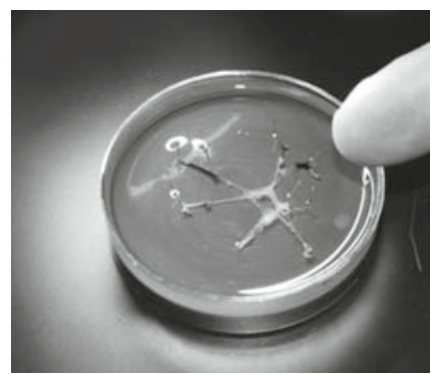

b.

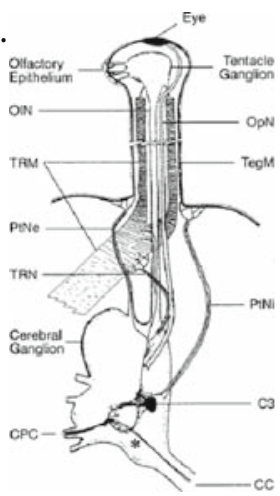

C.

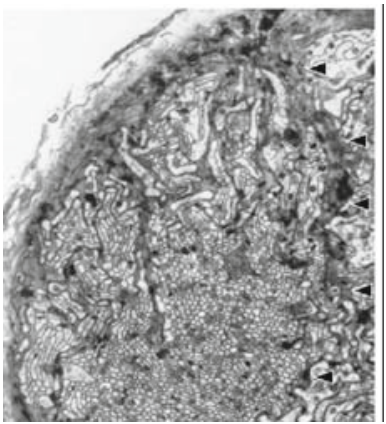

Fig. 25 Helix Aspersa morphological details. (a) dorsal view of circumoesophageal ring showing eye stalks. The black spots at the 10 o'clock and 2 o'clock positions are the eyes. Preparation is shown in a $30 \mathrm{~mm}$ Petri dish with the neural preparation pinned to a sylgard184 substrate. (b) diagrammatic cross section of the eye stalk, reprinted with permission from [159] (c) Cross section of the intestinal nerve, ovotestis branch [133]. The densely packed small $(0.2 \mu \mathrm{m}-0.5 \mu \mathrm{m})$ axons typical of $H$. Aspersa nerves are clearly seen

changes in external salt concentration or temperature because the preparations are robust. In both $H$. aspersa and $P$. clarkii oscillatory structures have been located that produce rhythmic repeatable patterns [140, 141]. These structures offer the opportunity to study subtle effects of magnetic fields on structures with a strong intrinsic behavior.

\section{Methods}

\subsection{Data Acquisition and Control}

While it is beyond the scope of this chapter to discuss an entire electrophysiology recording and stimulation system, we will include a few resources. It is probably best to learn techniques and about the equipment from users in an active lab. If this 
is not possible, several excellent resources exist. Details of construction of an electrophysiology station are reviewed in [142] and exhaustive details on methods of recording and stimulation are covered in [143, 144]. A computer-controlled data acquisition system is a must for magnetic stimulation systems. Computer control provides some measure of safety for the operator during discharge and enables for precise measurement. Exact timing information is required to determine when the magnetic stimulation pulse was generated and when and where the neural signal is generated. This requires tens of microseconds timing accuracy to correctly infer propagation velocity and thus the locus of excitation for small neural preparations. An excellent resource for data acquisition systems is [145].

Given time, equipment can be constructed in the lab or purchased second hand. An inexpensive recording amplifier described in [146] and stimulators are described in [147-149]. The current and voltage compliance of the stimulator increase significantly as the distance increases from the point source to the neural cable. Telescoping amplifier methods [150] are easily employed to extend the operating range of stimulators.

MATLAB has gained wide acceptance as a development platform for the acquisition and analysis of electrophysiological data. Notably among these tools are several free packages. Gprime is a free package for data acquisition and control that works with a wide range of National Instruments data acquisition cards, can perform rudimentary spike sorting, and supply a synchronized signal to a stimulator [151]. SigTool is a suite of processing tools for the analysis of neural data with a wide range of import capabilities for commercial data acquisition systems [152]. SigTool calls wave_clus to perform spike sorting and clustering and wave_clus is also a standalone analysis package [153]. Wave_clus has both tutorials and sample data available at http://www.vis.caltech.edu/ $\sim$ rodri/Wave_clus/Wave_clus_home.htm.

\subsubsection{Electrophysiological Recording and Stimulation}

To record electrophysiological signals, we employ both suction electrodes and custom designed recording chambers based on a cuff electrode recording apparatus. Suction electrode recordings from nerve trunks yield compound action potentials from a group of neurons, however, due to diameter-based recruitment the recorded CAP constitutes of spikes from only a small subgroup of the larger axons, while offering a relative ease of handling and robust experimental protocol. Suction electrodes can be easily constructed [149]. Significant improvements in recording stability and signal fidelity are possible with the use of glass suction electrodes made from smooth wall micropipette glass, flamed to form a smooth hourglass shape opening (as shown in Fig. 26). A successful recording will produce very stable, reproducible signals over several hours. We have been able to successfully apply spike sorting to extracellularly recorded action potentials using these suction electrodes.

The cuff electrode recording chamber is based on the apparatus presented in [154]. The chamber is constructed by tying fine gauge electrode wire around a 


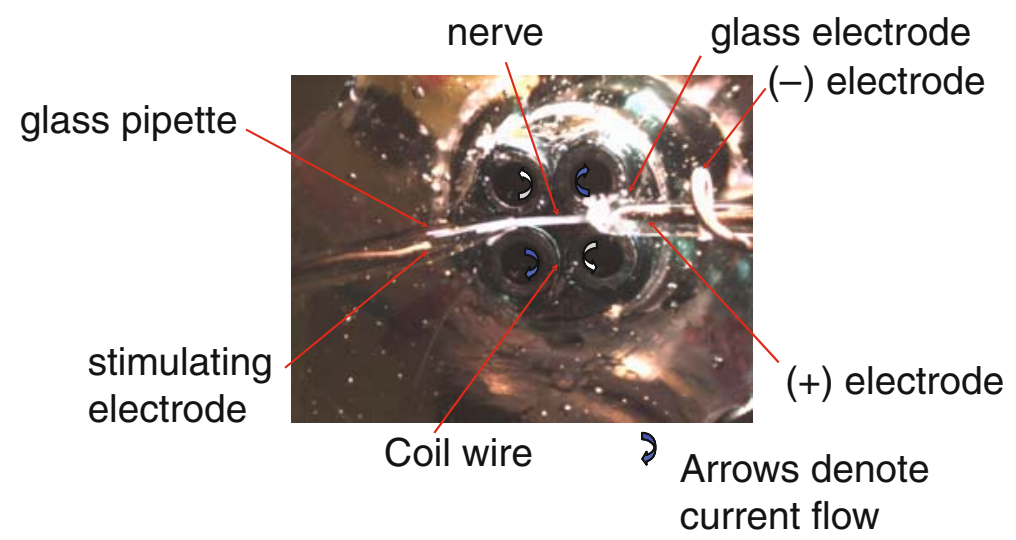

Fig. 26 Top view of magnetic stimulation and suction electrode rig

Teflon tube of the approximate diameter of the nerve under study. Teflon sleeving assortments are available from Sparky Electronics, Fresno, CA and Fry's Electronics, San Jose, CA 95112. Gold and silver are appropriate wires for the recording and a significant decrease in noise is observed when the silver wire is chlorided. Platinum wire can be stiff even at very fine gauge sizes. Fine gauge precious metal wire is available from A-M Systems, MWS Wire Industries, Westlake Village, CA 91362 and California Fine Wire, Grover Beach, CA 934830446 . The assembly is taped or pinned down over a sheet of polyimide roughened to facilitate adhesion. Insulted wires to interface with the recording amplifier are silver epoxied to the free end of the recording wire. The entire assembly is then covered with SYLGARD ${ }^{\circledR} 184$ (Dow Corning) silicone elastomer and cured. After curing, the apparatus is trimmed to size and the Teflon tube is removed. The resulting device is shown in Fig. 27. A hole is drilled in the bottom of a Petri dish and the whole apparatus glued to the bottom of the Petri dish. Since the KAPTON is a known thickness, and an excellent insulator, small nerves can placed with reasonable repeatability close to the coil.

In our set-up electrophysiological signals were recorded using glass suction electrodes filled with bath saline. Signals were amplified by an A-M Systems model 1700 amplifier (www.a-msystems.com) set at a gain of $1000 \times$ and hardware filtered at $10 \mathrm{~Hz}$ and $5 \mathrm{kHz}$. Axon Instruments pClamp (http://www.moleculardevices.com) was used for data collection and triggering of both magnetic and electrical pulse generators. Data were digitally filtered at $100 \mathrm{~Hz}$ and $5 \mathrm{kHz}$.

Electrical stimulation was performed using a S8800 Grass Technologies stimulator (http://www.grasstechnologies.com). A custom designed modified Howland current source triggered directly form the output of the Grass $\mathbf{S} 8800$ provided the current pulse to the preparation. Magnetic stimulation was accomplished using an Agilent 33120 A arbitrary waveform generator driving a high-power V-I converter or a thyristor triggered pulse discharge circuit. Further details on the circuits may 
Fig. 27 Modified nerve cuff recording apparatus.

(a) system diagram and recording hook up to amplifier. (b) end view

(c) top view

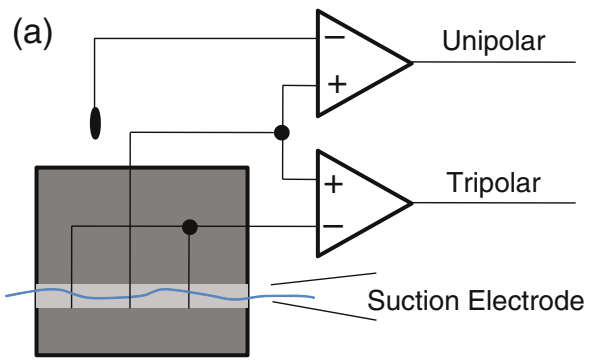

(b)

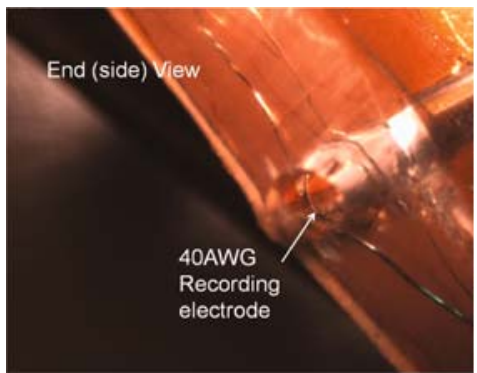

(c)

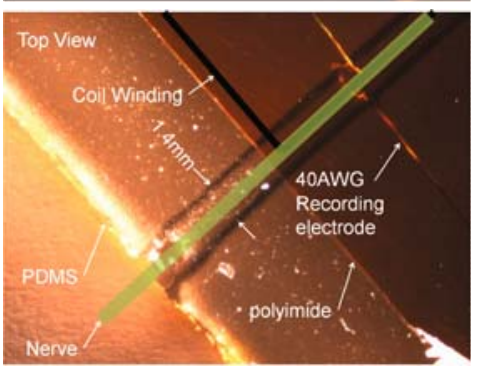

be obtained from [155]. Axon Instruments Clampfit was used for threshold windowing and peak alignment.

\subsubsection{Tissue Culture}

Snails can be obtained from biological supply houses. Adults with shells $2-3 \mathrm{~cm}$ in diameter are used for recording. Snails are fed a diet of cornmeal or bird seed and provided oyster shells as a calcium source. Light, maintained at $12 \mathrm{~h}$ cycles, can be inversed such that the snails are active during the day. Individual specimens were anesthetized with $2 \mathrm{ml}$ cold $\left(10^{\circ} \mathrm{C}\right) 50 \mathrm{mM} \mathrm{MgCl}$ and prepared as in [138]. The nerves in the eyestalk as well as the pedal nerves located on the ventral surface of the subesophageal ganglia are good targets for magnetic stimulation because of their length as well as readily recordable CAPs.

Crawfish adults are $7-11 \mathrm{~cm}$ in length. Specimens should be kept in individual tanks and maintained on a mixed diet of vegetable and protein matter. Dissection can be performed by anesthetizing the animal in an ice bath and cutting off the tail (abdomen). The abdomen is pinned ventral side up and bathed in crawdad 
saline prepared as in [138]. Swimmerets need to be removed and the cuticle cut along the sides of the carapace of the abdominal section. The cuticle can then be carefully removed, the abdominal nerve cord freed from the surrounding muscle tissue, and lifted into clean crawdad saline solution.

\section{Results}

A large shift in the chronaxy of the strength-duration curve would be expected from the difference in physiology and morphology between $H$. aspera and $P$. clarkii. As shown in Fig. 28, the chronaxy of $H$. aspersa and $P$. clarkii vary by approximately 20 times. The rheobase varied much less, by approximately two.

Initial testing results of magnetic stimulation appear promising. Figure 29(a and $b$ ) show magnetic stimulation of single action potentials using ferrite cores. For Fig. 29a, the core used was a $22 \mu \mathrm{H}$ coil stimulated with a linear saw tooth current ramp of $0.1 \mathrm{~A} / \mu \mathrm{s}$ for $250 \mu \mathrm{S}$. For Fig. 29b, the magnetic pulse was

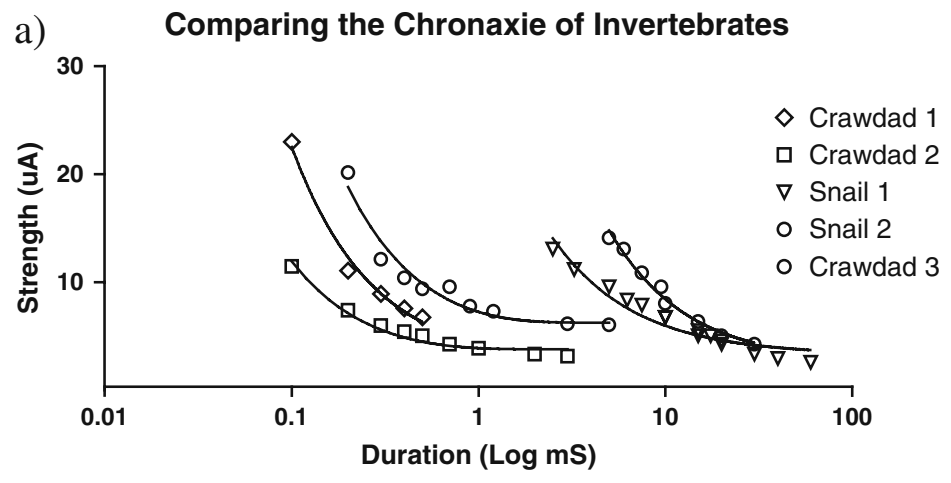

b) Comparing the Chronaxie of Invertebrates

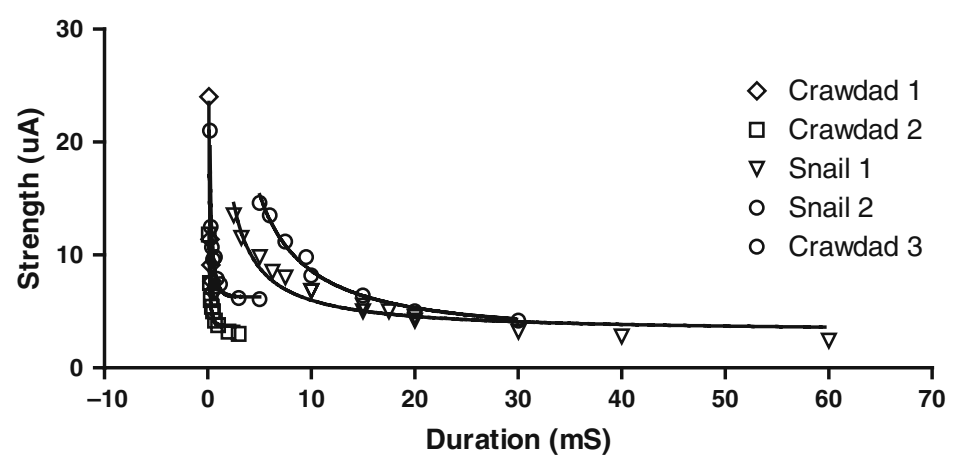

Fig. 28 Comparison of strength-duration curves of invertebrates. Experimentally derived strength-duration curves using electrical stimulation and suction electrodes. (a) shows a log-linear plot and (b) shows a linear-linear plot. Similar rheobase, but the chronaxy varies by as much as an order of magnitude. Curve fitting was performed using a non-linear fit to the exponential strength-duration equation 
a.

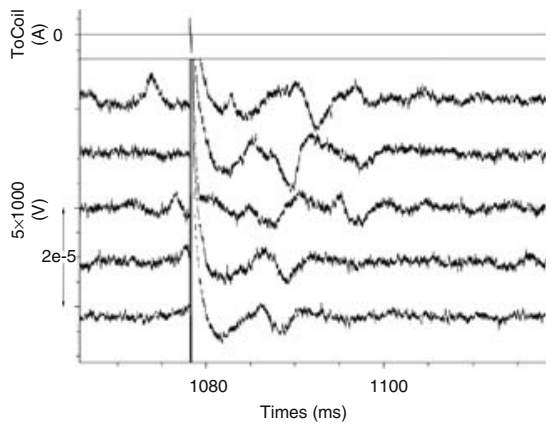

b.

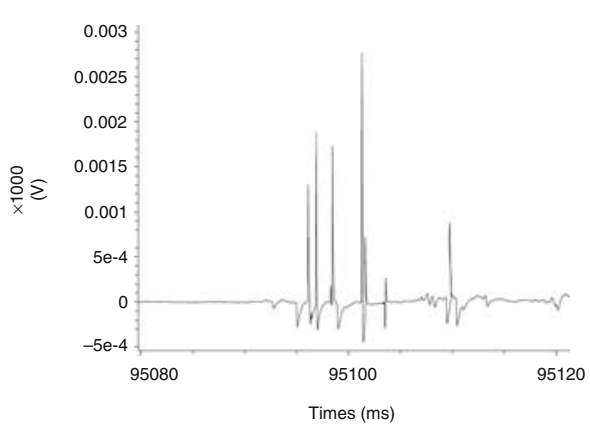

Fig. 29 Magnetically generated action potentials. (a) Single Action potential generated by magnetic stimulation with ferrite core using $H$. Aspersa neurobiological preparation. Upper trace is the current delivered to the coil. Lower 5 traces are recorded responses from repetitive sweeps. The stimulus artifact aligns with the spike in the upper trace. (b) set of individual action potentials generated by magnetic stimulation with ferrite core using P. Clarkii neurobiological preparation. Note the stimulus artifact to the left of the first action potential

a)
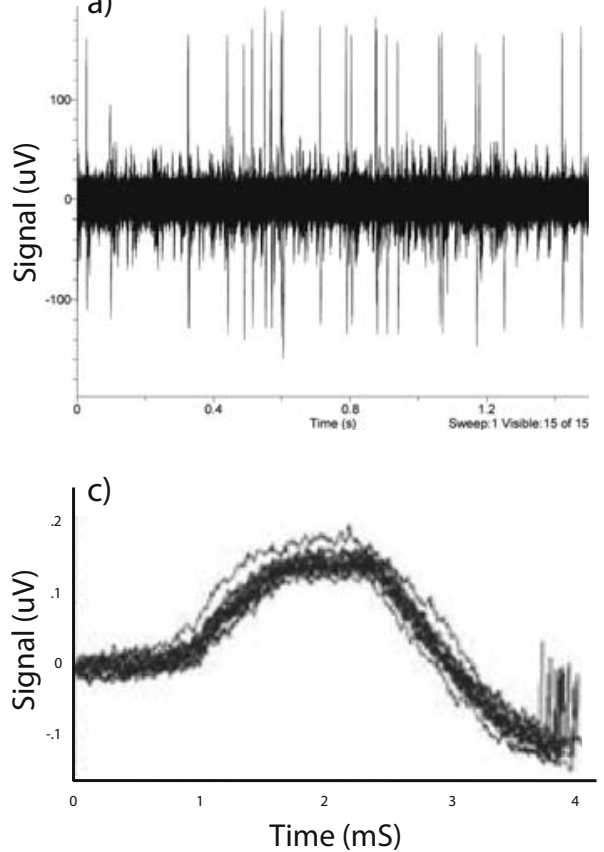

b)
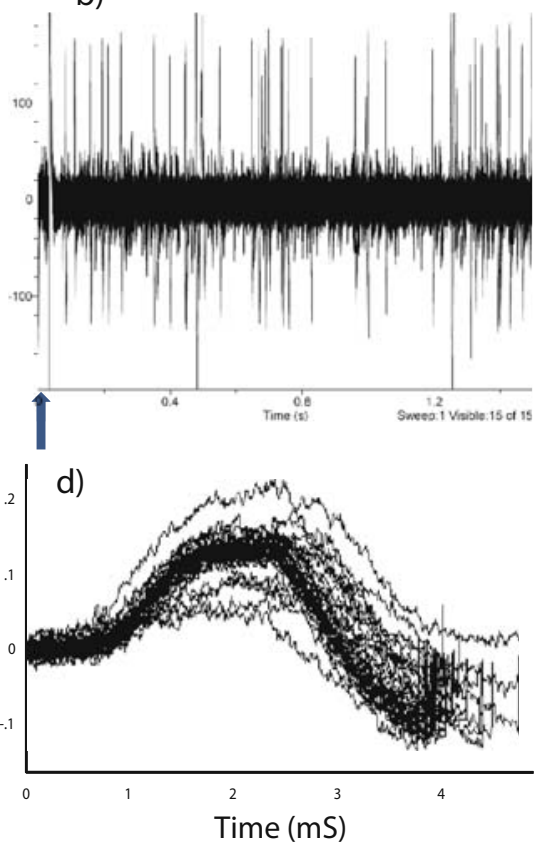

Fig. 30 Variation in spiking frequency and action potential shape induced by magnetic stimulation. (a) 19 neural spikes recorded without stimulus (b) 36 neural spikes recorded with stimulus (c) Aligned action potentials from (a) (d) Aligned action potentials from (b). Data is 15 sweeps 30 s apart. Arrow in (b) denotes magnetic stimulation stimulus artifact 
generated with a thyristor triggered circuit using a bank of $1000 \mu \mathrm{F}$ flash capacitors charged to $200 \mathrm{~V}$. The coil was a rectangular coil $2.2 \mathrm{~mm}$ by $4.4 \mathrm{~mm}$ of material 73 wound to an impedance value of $24 \mu \mathrm{H}$.

Subthreshold stimuli will not elicit an action potential but should alter the intrinsic behavior of oscillatory firing patterns. The effect of subthreshold magnetic stimulation alters both the firing frequency and the shape of the recorded action potentials in Fig. 30b and 30d. The change in spiking frequency and the variation of action potential shape are clear evidence of subthreshold interaction of magnetic fields with neural tissue.

\section{Conclusion}

Magnetic stimulation of neural tissue has a wide range of clinical applications. However, the future development of the field depends upon development of improved technologies and quantitative models are a part of this development. To further this goal we outlined a complete approach to developing small-scale magnetic stimulation experiments and showed how it could be applied to novel circuit topologies. The use of ferrite cores has allowed magnetic stimulation to be scaled to the point that a wide variety of neurobiological preparations are available for study. With new preparations comes new opportunities for investigation and understanding. Using a wide array of neurobiological preparations offers the opportunity to investigate the finer details of magnetic stimulation including magnetic stimulation nerve recruitment, blocking studies, and long-term histological effects.

Acknowledgment The authors would like to acknowledge Dr. David Tauck of Santa Clara University for his guidance during the dissections and electrophysiological experiments, Bennett Lee for his assistance with the neural preparations, Dr. David Parent for valuable technical discussion on coil and circuit design and numerical analysis, and Melissa Wibom for draft editing assistance. We would like to acknowledge the NSF for their funding support.

\section{Appendix: Modeling Magnetic Stimulation with Solenoid Coil}

The circular E field generated by a solenoid coil with core (shown in Fig. 31) can be approximated as

$$
E \approx\left\{\begin{array}{c}
\alpha \frac{r}{2} u_{r} u_{0} n \frac{d I}{d t}, r<\rho, z<<\rho \\
\alpha \frac{\rho^{2}}{2 r} u_{r} u_{0} n \frac{d I}{d t}, r>\rho, z<<\rho,
\end{array}\right.
$$


Fig. 31 Illustration of magnetic field flux generated by long sinusoid coil

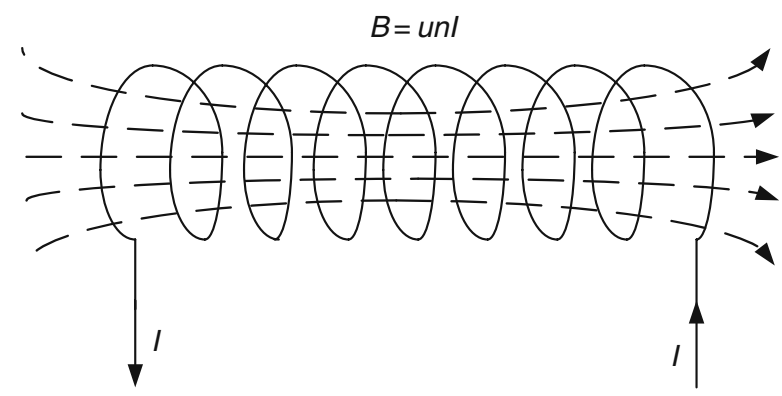

where $\alpha(0<\alpha<1)$ is a parameter to compensate the distance between the coil and the neural tissue, $\rho$ denotes the radius of the inductor, and $r$ represents the distance from the point of interest to the center of the current loop.

Within the circular loop, the E field derivative can be evaluated in $x-y$ coordinates as

$$
E=E_{x} \hat{x}+E_{y} \hat{y}=-\frac{y}{2} \alpha u_{r} u_{0} n \frac{d I}{d t} \hat{x}+\frac{x}{2} \alpha u_{r} u_{0} n \frac{d I}{d t} \hat{y}
$$

From Equation (55), the spatial derivative of the E field is almost zero, given $r \leq \rho$

$$
\left\{\begin{array}{l}
\frac{\partial E_{x}}{\partial x}=-\frac{\partial \alpha \frac{y}{2} u_{r} u_{0} n \frac{d I}{d t}}{\partial x}=0, r<\rho, z<<\rho \\
\frac{\partial E_{y}}{\partial y}=\frac{\partial \alpha \frac{x}{2} u_{r} u_{0} n \frac{d I}{d t}}{\partial y}=0, r<\rho, z<<\rho .
\end{array}\right.
$$

Equation (56) shows that a neuron directly above a solenoid coil is difficult to stimulate. This property is irrelevant to the geometry variation of neurons and it indicates potentially improved spatial selectivity compared with functional electric stimulation, where neurons close to the electrode are all stimulated.

If a part of the neuron or the whole neuron is located outside the current loop (shown in Fig. 32, the right upper neuron), the E field derivative can be derived from (54) as

$$
E=E_{x} \hat{x}+E_{y} \hat{y}=-\alpha \frac{y}{2} \frac{\rho^{2}}{r^{2}} u_{r} u_{0} n \frac{d I}{d t} \hat{x}+\alpha \frac{x}{2} \frac{\rho^{2}}{r^{2}} u_{r} u_{0} n \frac{d I}{d t} \hat{y} .
$$


Fig. 32 Illustration of nerve location and orientation referred to the stimulating coil that is indicated by the circle

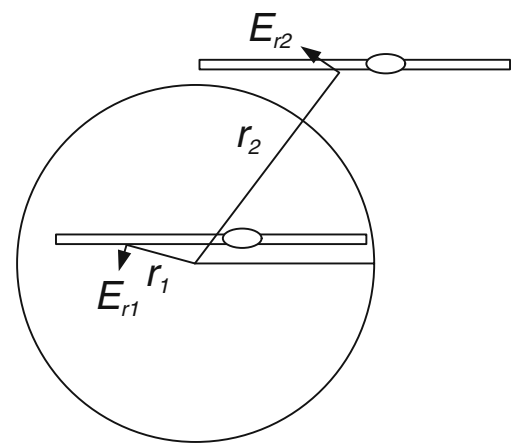

In this case, the spatial derivative of the E field is non-zero, as $\mathrm{r}$ is a function of both $x$ and $y$, thus

$$
\left\{\begin{array}{l}
\frac{\partial E_{x}}{\partial x}=-\frac{\partial \alpha \frac{y}{2} \frac{\rho^{2}}{r^{2}} u_{r} u_{0} n \frac{d I}{d t}}{\partial x}=-\frac{y}{2} \alpha \rho^{2} u_{r} u_{0} n \frac{d I}{d t} \frac{\partial r^{-2}}{\partial x}=\alpha \rho^{2} u_{r} u_{0} n \frac{d I}{d t} \frac{x y}{r^{4}}, r>\rho, z<<\rho \\
\frac{\partial E_{y}}{\partial y}=\frac{\partial \alpha \frac{x}{2} \frac{\rho}{r^{2}} u_{r} u_{0} n \frac{d I}{d t}}{\partial y}=\frac{x}{2} \alpha \rho^{2} u_{r} u_{0} n \frac{d I}{d t} \frac{\partial r^{-2}}{\partial y}=-\alpha \rho^{2} u_{r} u_{0} n \frac{d I}{d t} \frac{x y}{r^{4}}, r>\rho, z<<\rho
\end{array}\right.
$$

According to Equation (58), the maximum spatial derivative is obtained when

$$
|x|=|y|=\frac{\sqrt{2}}{2} \rho
$$

The maximum spatial derivative of the E field over both $x$ and $y$ axes are

$$
\left|\frac{\partial E_{x}}{\partial x}\right|=\left|\frac{\partial E_{y}}{\partial y}\right|=\frac{1}{2} \alpha u_{r} u_{0} n \frac{d I}{d t} .
$$

Equations (58) and (59) suggest an optimal coil-axon configuration, this shown in Fig. 33. To achieve, two conditions should be satisfied: (1) the membrane segment should be close to the current loop and (2) the axon's direction is approximately $45^{\circ}$ deviated from the radius.

Other configurations, e.g., neuron inside the coil radius (Fig. 32), or the axon crossing the coil center (Fig. 34a), or neuron completely outside the coil radius (Fig. 34b), introduce a smaller E field derivative compared with the optimal configuration. In all cases, the spatial derivative of $\mathrm{E}$ field can be evaluated using Equation (58). 
Fig. 33 Optimal nerve location and orientation that maximizes the induced E field derivative

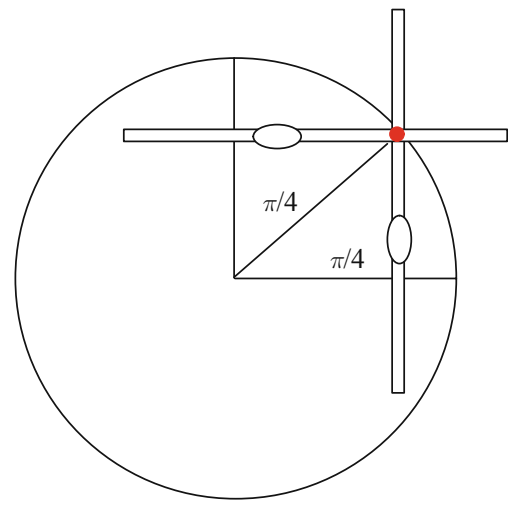

Fig. 34 (a) Not excitable coil nerve geoemtry Configuration. (b) excitable, but less preferreable configuration compared with Fig. 33.

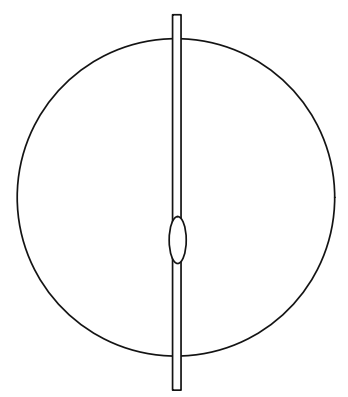

(a)

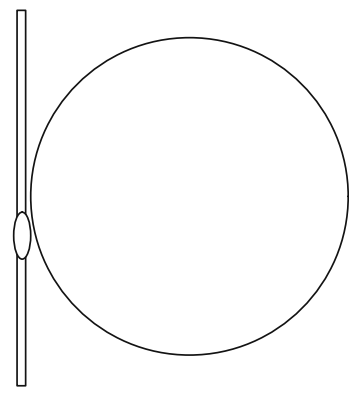

(b)

\section{Current Slew Rate and Power Consumption}

\subsection{Slew Rate}

A magnetically induced spatially varying $E$ field of about $10 \mathrm{kV} / \mathrm{m}^{2}$ is required for a duration near the chronaxy of the nerve to stimulate neurons [59, 156, 157]. Given a conventional air-core coil with diameter on the order of a few $\mathrm{cm}$, a current slew rate greater than $10 \mathrm{~A} / \mathrm{us}$ is required for excitation of neural tissue placed with a few $\mathrm{mm}$. According to Equation (59), the maximum spatial derivative of $\mathrm{E}$ field generated by a solenoid coil is 
Fig. 35 Illustration of magnetic field flux generated by a current loop or $\mathrm{N}$ superimposed current loops

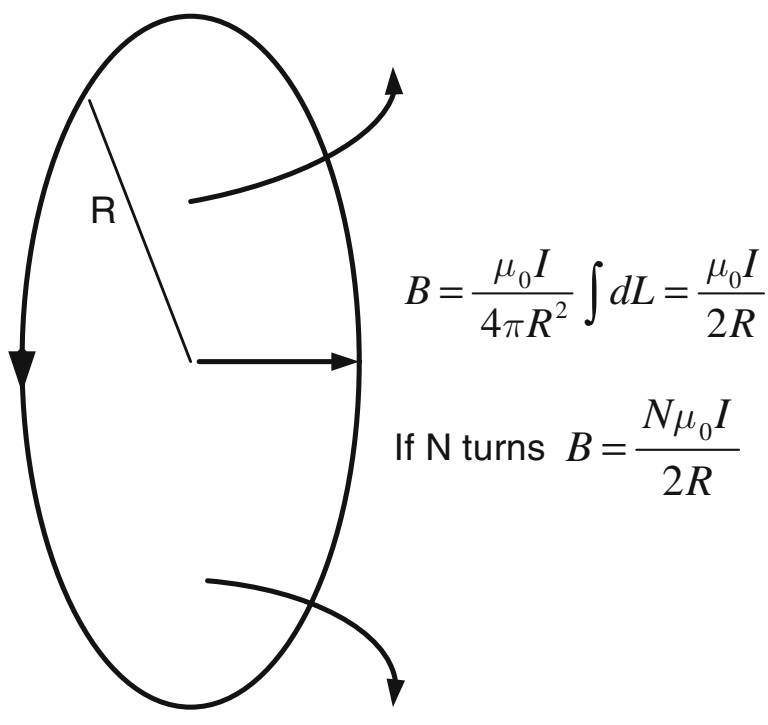

$$
\frac{1}{2} \alpha u_{r} u_{0} n \frac{d I}{d t}
$$

Given $u_{0}=4 \pi \times 10^{-7}$ and the required current slew rate is

$$
{\frac{d I}{d t_{\text {Threshold }}}}=1.6 \times 10^{10} \frac{L}{\alpha \mu_{r} N}
$$

where $\mathrm{N}$ is the number of turns, $\mathrm{L}$ is the coil height and

$$
\frac{d E}{d x}_{\text {Threshold }}=10^{10} \mathrm{~V} / \mathrm{m}^{2}
$$

Using values reasonable to implement experimentally; $\mathrm{N}=100, \mu_{r}=20$, $\alpha=0.5$, and $\mathrm{L}=1 \mathrm{~cm}$, we have

$$
\frac{d I}{d t} \text { Threshold }=1.6 \times 10^{5} \mathrm{~A} / \mathrm{S}=0.16 \mathrm{~A} / \mu \mathrm{S} .
$$

According to Equation (59) and numeric examples shown in Equations (60) and (61), the loop diameter of a solenoid coil does not influence the required current slew rate to activate neurons. 


\subsection{Power Consumption}

The required energy per pulse to active a neuron is

$$
\int 0.5 I^{2} R_{\text {res }} d t=\int 0.5\left(\frac{d I}{d t} \text { Threshold } t\right)^{2} R_{\text {res }} d t=0.17 \frac{d I^{2}}{d t}{ }_{\text {Threshold }} R_{\text {res }} T^{3}
$$

where $T$ is the current pulse width and $\mathrm{R}_{\text {res }}$ is the coil resistance. For a $100 \mu \mathrm{S}$ pulse with a $1 \Omega$ coil resistance, the dissipated energy is only $4 \mathrm{~mJ}$. The technique of using biphasic sawtooth waveform can further reduce power dissipation by half.

\section{Scaling with Planar Coil}

Similar to Equation (54), the E field generated by a circular planar coil is approximated as

$$
E \approx\left\{\begin{array}{c}
\alpha \frac{r}{2 \rho} u_{r} u_{0} N \frac{d I}{d t}, r<\rho, z<<\rho \\
\alpha \frac{\rho}{2 r} u_{r} u_{0} N \frac{d I}{d t}, r>\rho, z<<\rho
\end{array}\right.
$$

For Case I, where $r<\rho$, following Equation (63), neurons inside the coil radius are less excitable. It is important to note that Equation (63) is approximate and a small E field spatial derivative still exists.

For Case II, where $r>\rho$, the field is approximated as

$$
E=E_{x} \hat{x}+E_{y} \hat{y}=-\alpha \frac{y}{2} \frac{\rho}{r^{2}} u_{r} u_{0} N \frac{d I}{d t} \hat{x}+\alpha \frac{x}{2} \frac{\rho}{r^{2}} u_{r} u_{0} N \frac{d I}{d t} \hat{y}
$$

where the spatial derivative of E field can be obtained from Equation (64) as

$$
\left\{\begin{array}{l}
\frac{\partial E_{x}}{\partial x}=-\frac{\partial \alpha \frac{y}{2} \frac{\rho}{r^{2}} u_{r} u_{0} N \frac{d I}{d t}}{\partial x}=-\alpha \frac{y}{2} \rho u_{r} u_{0} N \frac{d I}{d t} \frac{\partial r^{-2}}{\partial x}=\alpha \rho u_{r} u_{0} N \frac{d I}{d t} \frac{x y}{r^{4}}, r>\rho, z<<\rho \\
\frac{\partial E_{y}}{\partial y}=\frac{\partial \alpha \frac{x}{2} \frac{\rho}{r^{2}} u_{r} u_{0} N \frac{d I}{d t}}{\partial y}=\alpha \frac{x}{2} \rho u_{r} u_{0} N \frac{d I}{d t} \frac{\partial r^{-2}}{\partial y}=-\alpha \rho u_{r} u_{0} N \frac{d I}{d t} \frac{x y}{r^{4}}, r>\rho, z<<\rho
\end{array}\right.
$$

The maximum E field spatial derivative is achieved when $|x|=|y|=\frac{\sqrt{2}}{2} \rho$ 


$$
\left|\frac{\partial E_{x}}{\partial x}\right|=\left|\frac{\partial E_{y}}{\partial y}\right|=\frac{1}{2} u_{r} u_{0} \frac{N}{\alpha \rho} \frac{d I}{d t}
$$

Comparing Equation (66) to (59), the spatial derivative is related to the loop radius $\rho$. With planar coils, reducing the loop radius can increase the $\mathrm{E}$ field spatial derivative. A few numeric examples are included below to describe the scaling effect.

Case A: 20 turns, $\rho=4 \mathrm{~cm}$ and $u_{r}=1$, the required minimal current slew rate is

$$
\frac{d I}{d t}=3 \times 10^{7} \mathrm{~A} / \mathrm{S}=30 \mathrm{~A} / \mu \mathrm{S}
$$

Case B: 20 turns, $\rho=4 \mathrm{~mm}$ and $u_{r}=1$, the required minimal current slew rate is

$$
\frac{d I}{d t}=3 \times 10^{6} A / S=3 A / \mu S
$$

\section{References}

1. Weintraub, M.I. and S.P. Cole, Pulsed magnetic field therapy in refractory neuropathic pain secondary to peripheral neuropathy: electrodiagnostic parameters - pilot study. Neurorehabilitation and Neural Repair, 2004, 18(1): 42.

2. Pujol, J., et al., The effect of repetitive magnetic stimulation on localized musculoskeletal pain. Neuroreport, 1998, 9(8): 1745-1748.

3. Basser, P.J. and B.J. Roth, New currents in electrical stimulation of excitable tissues. Annual Review of Biomedical Engineering, 2000, 2(1): 377-397.

4. Barker, A.T., The history and basic principles of magnetic nerve stimulation. Electroencephalogr Clin Neurophysiol Suppl, 1999, 51: 3-21.

5. Geddes, L.A., History of magnetic stimulation of the nervous system. J Clin Neurophysiol, 1991, 8(1): 3-9.

6. Hallett, M., Transcranial magnetic stimulation and the human brain. Nature, 2000, 406(6792): 147-150.

7. George, M.S., et al., Transcranial magnetic stimulation. Neurosurgery Clinics of North America, 2003, 14(2): 283-301.

8. Hallett, M., Transcranial magnetic stimulation: a primer. Neuron, 2007, 55(2): 187-199.

9. Kato, M., Electromagnetics in biology. 2006, Tokyo: Springer. xv, 324p.

10. Ji, R.-R., et al., Repetitive transcranial magnetic stimulation activates specific regions in rat brain. Proceedings of the National Academy of Sciences of the United States of America, 1998, 95(26): 15635-15640.

11. Li, G.N. and D. Hoffman-Kim, Tissue-engineered platforms of axon guidance. Tissue Engineering Part B: Reviews, 2008, 14(1): 33-51.

12. Eaton, H.A.C. The electric field induced in a spherical volume conductor by a Magnetic Coil. in Engineering in Medicine and Biology Society, 1990, Proceedings of the Twelfth Annual International Conference of the IEEE. 1990.

13. Bencsik, M., R. Bowtell, and R.M. Bowley, Electric fields induced in a spherical volume conductor by temporally varying magnetic field gradients. Physics in Medicine and Biology, 2002(4): 557-576. 
14. Ravazzani, P., et al., Magnetic stimulation of the nervous system: Induced electric field in unbounded, semi-infinite, spherical, and cylindrical media. Annals of Biomedical Engineering, 1996, 24(5): 606-616.

15. Roth, B.J. and P.J. Basser, A model of the stimulation of a nerve fiber by electromagnetic induction. Biomedical Engineering, IEEE Transactions on, 1990, 37(6): 588-597.

16. Miranda, P.C., et al., Tissue heterogeneity as a mechanism for localized neural stimulation by applied electric fields. Physics in Medicine and Biology, 2007, 52(18): 5603-5617.

17. Ye, H., M. Cotic, and P.L. Carlen, Transmembrane potential induced in a spherical cell model under low-frequency magnetic stimulation. Journal of Neural Engineering, 2007, 4(3): 283-293.

18. Rotem, A. and E. Moses, Magnetic stimulation of curved nerves. Biomedical Engineering, IEEE Transactions on, 2006, 53(3): 414-420.

19. Rotem, A. and E. Moses, Magnetic stimulation of one-dimensional neuronal cultures. Biophysical Journal, 2008.

20. References, S., et al., Magnetic coil stimulation of straight and bent amphibian and mammalian peripheral nerve in vitro: locus of excitation. Journal of Physiology, 1993, 460: 201-219.

21. Hsu, K.H., S.S. Nagarajan, and D.M. Durand, Analysis of efficiency of magnetic stimulation. Biomedical Engineering, IEEE Transactions on, 2003, 50(11): 1276-1285.

22. Engstrom, S. and R. Fitzsimmons, Five hypotheses to examine the nature of magnetic field transduction in biological systems. Bioelectromagnetics, 1999, 20(7): 423-430.

23. Struijk, J.J. and V. Schnabel, Difference between electrical and magnetic nerve stimulation: a case for the transverse field? Engineering in Medicine and Biology Society, 2001. Proceedings of the 23rd Annual International Conference of the IEEE, 2001, 1.

24. Wasserman, E., C.M. Epstein, and U. Ziemann, The Oxford handbook of transcranial stimulation. 2008, Oxford; New York: Oxford University Press. xiv, 747p.

25. Ridding, M.C. and J.C. Rothwell, Is there a future for therapeutic use of transcranial magnetic stimulation? Nature Reviews and Neuroscience, 2007, 8(7): 559-567.

26. Hodgkin, A.L. and W.A.H. Rushton, The electrical constants of a crustacean nerve fibre. Proceedings of the Royal Society of London. Series B, Biological Sciences (1934-1990), 1946, 133(873): 444-479.

27. Ranck, J.B., Which elements are excited in electrical stimulation of mammalian central nervous system: a review. Brain Research, 1975, 98(3): 417-440.

28. Patterson, M.M. and R.P. Kesner, Electrical stimulation research techniques. Methods in physiological psychology, 1981. New York: Academic Press. xv, 370p.

29. Rattay, F., Ways to approximate current-distance relations for electrically stimulated fibers. Journal of Theoretical Biology, 1987, 125(3): 339-349.

30. Rattay, F., Analysis of models for extracellular fiber stimulation. Biomedical Engineering, IEEE Transactions on, 1989, 36(7): 676-682.

31. Roth, B.J., Mechanisms for electrical stimulation of excitable tissue. Crit Rev Biomed Eng, 1994, 22(3-4): 253-305.

32. Plonsey, R. and R.C. Barr, Bioelectricity: a quantitative approach. 3rd ed. 2007, New York: Springer. xiv, 528p.

33. Schnabel, V. and J.J. Struijk, Evaluation of the cable model for electrical stimulation of unmyelinated nerve fibers. Biomedical Engineering, IEEE Transactions on 2001, 48(9): $1027-1033$.

34. Zierhofer, C.M. and C.M. Zierhofer, Analysis of a linear model for electrical stimulation of axons-critical remarks on the "activating function concept". Biomedical Engineering, IEEE Transactions on, 2001, 48(2): 173-184.

35. Hui, Y., et al. A New Model and improved cable function for representing the activating peripheral nerves by a transverse electric field during magnetic stimulation. In Neural Engineering, 2005. Conference Proceedings. 2nd International IEEE EMBS Conference on. 2005.

36. Nagarajan, S.S., S.S. Nagarajan, and D.M. Durand, A generalized cable equation for magnetic stimulation of axons. Biomedical Engineering, IEEE Transactions on, 1996, 43(3): 304-312. 
37. Struijk, J.J. and D.M. Durand. Magnetic peripheral nerve stimulation: axial versus transverse fields. in [Engineering in Medicine and Biology, 1999. 21st Annual Conf. and the 1999 Annual Fall Meeting of the Biomedical Engineering Soc.] BMES/EMBS Conference, 1999. Proceedings of the First Joint. 1999.

38. Hsu, K.H. and D.M. Durand, Prediction of neural excitation during magnetic stimulation usingpassive cable models. Biomedical Engineering, IEEE Transactions on, 2000, 47(4): 463-471.

39. Kai-Hsiung, H. and D.M. Durand. Determination of excitation thresholds of nerve fibers during magnetic stimulation. in Engineering in Medicine and Biology Society, 1996. Bridging Disciplines for Biomedicine. Proceedings of the 18th Annual International Conference of the IEEE. 1996.

40. Suarez-Antola, R.E. The time constants for the electric stimulation of nerve an muscle fibers by point electrodes. in Neural Engineering, 2005. Conference Proceedings. 2nd International IEEE EMBS Conference on. 2005.

41. Malmivuo, J. and R. Plonsey, Bioelectromagnetism: principles and applications of bioelectric and biomagnetic fields. 1995, New York: Oxford University Press. xxii, 482.

42. Irnich, W., Electrostimulation by time-varying magnetic fields. Magnetic Resonance Materials in Physics, Biology and Medicine, 1994, 2(1): 43-49.

43. Ruohonen, J., et al., Transverse-field activation mechanism in magnetic stimulation of peripheral nerves. Electroencephalography and Clinical Neurophysiology/Electromyography and Motor Control, 1996, 101(2): 167-174.

44. Roth, B.J., B.J. Roth, and P.J. Basser, A model of the stimulation of a nerve fiber by electromagnetic induction. Biomedical Engineering, IEEE Transactions on, 1990, 37(6): 588-597.

45. Carnevale, N.T. and M.L. Hines, The Neuron book. 2006, Cambridge, UK; New York: Cambridge University Press. xix, 457p.

46. Bower, J.M. and D. Beeman, The book of Genesis: exploring realistic neural models with the GEneral NEural SImulation System. 1998. Springer-Verlag New York, Inc. New York.

47. Zeng, L. and M.D. Dominique, Extracellular voltage profile for reversing the recruitment order of peripheral nerve stimulation: a simulation study. Journal of Neural Engineering, 2004, 4: 202.

48. Brauer, J.R., Magnetic actuators and sensors. 2006, Hoboken, NJ: Wiley. xii, 308p.

49. Claycomb, J.R., Applied electromagnetics using QuickField \& MATLAB. 2008, Hingham, Mass: Infinity Science Press.

50. Lonngren, K.E., S.V. Savov, and R.J. Jost, Fundamentals of electromagnetics with MATLAB. 2007, Raleigh, NC: SciTech Pub. xx, 528p.

51. Davey, K., L. Luo, and D.A. Ross, Toward functional magnetic stimulation (FMS) theory and experiment. Biomedical Engineering, IEEE Transactions on, 1994, 41(11): 1024-1030.

52. Nagarajan, S.S., D.M. Durand, and E.N. Warman, Effects of induced electric fields on finite neuronal structures: a simulation study. Biomedical Engineering, IEEE Transactions on, 1993, 40(11): 1175-1188.

53. Durand, D., A.S. Ferguson, and T. Dalbasti, Effect of surface boundary on neuronal magnetic stimulation. Biomedical Engineering, IEEE Transactions on, 1992, 39(1): 58-64.

54. Davey, K. and M. Riehl, Designing transcranial magnetic stimulation systems. Magnetics, IEEE Transactions on, 2005, 41(3): 1142-1148.

55. Ren, L. and S. Ueno, Calculating the activating function of nerve excitation in inhomogeneous volume conductor during magnetic stimulation using the finite element method. Magnetics, IEEE Transactions on, 2000, 36(4): 1796-1799.

56. Mathis, J., et al., The boundary effect in magnetic stimulation. Analysis at the peripheral nerve. Electroencephalography and Clinical Neurophysiology/Electromyography and Motor Control, 1995, 97(5): 238-245.

57. Esselle, K.P. and M.A. Stuchly, Neural stimulation with magnetic fields: analysis of induced electric fields. Biomedical Engineering, IEEE Transactions on, 1992, 39(7): 693-700. 
58. Olree, K.S. and K.W. Horch, Differential activation and block of peripheral nerve fibers by magnetic fields. Muscle and Nerve, 2006, 34(2): 189-196.

59. Alkhateeb, A. and R.P. Gaumond. Excitation of frog sciatic nerve using pulsed magnetic fields effect of waveform variations. in Engineering in Medicine and Biology Society, 1995, IEEE 17th Annual Conference. 1995.

60. Sommers, J.D. and R.P. Gaumond. Spacing analysis of a focal magnetic stimulating coil. 1997.

61. Wheeler, H.A., Inductance formulas for circular and square coils. Proceedings of the Ieee, 1982, 70(12): 1449-1450.

62. Mohan, S.S., et al., Simple accurate expressions for planar spiral inductances. Ieee Journal of Solid-State Circuits, 1999, 34(10): 1419-1424.

63. Lontis, E.R., M. Voigt, and J.J. Struijk, Focality assessment in transcranial magnetic stimulation with double and cone coils. Journal of Clinical Neurophysiology, 2006, 23(5): 463.

64. Peterchev, A.V., R. Jalinous, and S.H. Lisanby, A transcranial magnetic stimulator inducing near-rectangular pulses with controllable pulse width (cTMS). Biomedical Engineering, IEEE Transactions on, 2008, 55(1): 257-266.

65. Bronzino, J.D., A. Zangen, Y. Roth, B. Voller, and M. Hallett, "Transcranial magnetic stimulation of deep brain regions: evidence for efficacy of the H-coil" in The biomedical engineering handbook. 3rd ed. The electrical engineering handbook series. 2006, Boca Raton: CRC/Taylor and Francis.

66. Ren, C., P.P. Tarjan, and D.B. Popovic, A novel electric design for electromagnetic stimulation-the Slinkycoil. Biomedical Engineering, IEEE Transactions on, 1995, 42(9): 918-925.

67. Han, B., et al., Some technical aspects of magnetic stimulation coil design with the ferromagnetic effect. Medical and Biological Engineering and Computing, 2003, 41(5): 516-518.

68. Davey, K. and C.M. Epstein, Magnetic stimulation coil and circuit design. Biomedical Engineering, IEEE Transactions on, 2000, 47(11): 1493-1499.

69. Han, B.H., et al., Some technical aspects of magnetic stimulation coil design with the ferromagnetic effect. Medical and Biological Engineering and Computing, 2003, 41(5): 516-518.

70. Epstein, C.M. and K.R. Davey, Iron-core coils for transcranial magnetic stimulation. Journal of Clinical Neurophysiology, 2002, 19(4): 376.

71. Salvador, R., et al., High-permeability core coils for transcranial magnetic stimulation of deep brain regions. In Engineering in Medicine and Biology Society, 2007. EMBS 2007. 29th Annual International Conference of the IEEE. 2007.

72. Åke Öberg, P., Magnetic stimulation of nerve tissue. Medical and Biological Engineering and Computing, 1973, 11(1): 55-64.

73. Maass, J. and M. Asa, Contactless nerve stimulation and signal detection by inductive transducer. Magnetics, IEEE Transactions on, 1970, 6(2): 322-326.

74. Ueno, S., P. Lövsund, and P. Öberg, Effect of time-varying magnetic fields on the action potential in lobster giant axon. Medical and Biological Engineering and Computing, 1986, 24(5): 521-526.

75. Ueno, S., et al., Capacitive stimulatory effect in magnetic stimulation of nerve tissue. Magnetics, IEEE Transactions on, 1978, 14(5): 958-960.

76. van den Bossche, A., Inductors and transformers for power electronics. 2005. Boca Raton, FL: CRC Press.

77. McLyman, C.W.T., W.T. McLyman, and M. McLyman, Transformer and inductor design handbook. 2004. Boca Raton, FL: CRC Press.

78. Van den Bossche, A., V. Valchev, and T. Filchev. Improved approximation for fringing permeances in gapped inductors. in Industry Applications Conference, 2002, 37th IAS Annual Meeting. Conference Record of the. 2002.

79. Zhi, Y., L. Wentai, and E. Basham, Inductor modeling in wireless links for implantable electronics. Magnetics, IEEE Transactions on, 2007, 43(10): 3851-3860. 
80. Brauer, J.R. and I.D. Mayergoyz, Finite-element computation of nonlinear magnetic diffusion and its effects when coupled to electrical, mechanical, and hydraulic systems. Magnetics, IEEE Transactions on, 2004, 40(2): 537-540.

81. Venkatachalam, K., et al., Accurate prediction of ferrite core loss with nonsinusoidal waveforms using only Steinmetz parameters. Computers in Power Electronics, 2002, Proceedings. 2002 IEEE Workshop on, 2002: 36-41.

82. Jieli, L., et al. Improved calculation of core loss with nonsinusoidal waveforms. In Industry Applications Conference, 2001, Thirty-Sixth IAS Annual Meeting. Conference Record of the 2001 IEEE. 2001.

83. Hurley, W.G., E. Gath, and J.G. Breslin, Optimizing the AC resistance of multilayer transformer windings with arbitrary current waveforms. Power Electronics, IEEE Transactions on, 2000, 15(2): 369-376.

84. Travis, B., IGBTs and MOSFETs vie for applications. EDN, 1999, 44(9): 77-88.

85. Burke, A., Ultracapacitors: why, how, and where is the technology. Journal of Power Sources, 2000, 91(1): 37-50.

86. Slenes, K.M., et al., Pulse power capability of high energy density capacitors based on anew dielectric material. Magnetics, IEEE Transactions on, 2001, 37(1 Part 1): 324-327.

87. Prutchi, D. and M. Norris, Design and development of medical electronic instrumentation: a practical perspective of the design, construction, and test of medical devices. 2005, Hoboken, NJ: Wiley-Interscience, $\mathrm{xv}, 461 \mathrm{p}$.

88. Pascual-Leone, A., Handbook of transcranial magnetic stimulation. 2002, New York: Arnold; Oxford University Press [distributor], x, 406p.

89. Webster, J.G. and J.W. Clark, Medical instrumentation : application and design. 3rd ed. 1998, New York: Wiley, xix, 691 p.

90. Geddes, L.A., Accuracy limitations of chronaxie values. Biomedical Engineering, IEEE Transactions on, 2004, 51(1): 176-181.

91. Beecroft, S.I., A. Alkhateeb, and R. Gaumond. Nerve fiber stimulation threshold for multiphase stimuli. In Bioengineering Conference, 1994. Proceedings of the 1994 20th Annual Northeast. 1994.

92. Pressman, A.I., Switching power supply design. 2nd ed. 1998, New York: McGraw-Hill, xviii, 682p.

93. Han, B.H., et al. Development of four-channel magnetic nerve stimulator. in Engineering in Medicine and Biology Society, 2001. Proceedings of the 23rd Annual International Conference of the IEEE. 2001.

94. Polson, M.J., A.T. Barker, and I.L. Freeston, Stimulation of nerve trunks with timevarying magnetic fields. Med Biol Eng Comput, 1982, 20(2): 243-244.

95. Guide, C.D.A., Application guide, snubber capacitors. http://www.cde.com/catalogs/ igbtAPPguide.pdf.

96. Severns, R., Design of snubbers for power circuits. Cornell Dublier Technical Papers, online at http://www.cde.com/tech/design.pdf.

97. Whites, K., RL Circuits, inductive kick, diode snubbers, lecture 4 EE322. http://whites. sdsmt.edu/classes/ee322/class_notes/322Lecture4.pdf, 2006.

98. Motto, E., Hybrid circuits simplify IGBT modul gate drive, in PCIM. 1999. Europe.

99. Kratz, R. and P. Wyder, Principles of pulsed magnet design. Engineering materials. 2002, Berlin; New York: Springer, ix, 181p.

100. Smith, P.W., Transient electronics: pulsed circuit technology. 2002, New York: John Wiley, xi, $272 \mathrm{p}$.

101. Perreault, D. and S. Mogren, Magnetic Stimulator Power and Control Circuit, R.B. CArr (ed.) USPTO, 2003, Engineering, Onc: USA.

102. Basser, P.J., P.J. Basser, and B.J. Roth. Scaling laws for electromagnetic stimulation of an axon. In Engineering in Medicine and Biology Society, 1990. Proceedings of the Twelfth Annual International Conference of the IEEE. 1990. 
103. Fink, D.G. and H.W. Beaty, Standard handbook for electrical engineers. 1999, New York: McGraw-Hill., p. v.

104. Mesut, S. and T. Yanmei, Non-rectangular waveforms for neural stimulation with practical electrodes. Journal of Neural Engineering, 2007, 3: 227.

105. Wada, S., et al., Effects of stimulus waveform on magnetic nerve stimulation. Japanese Journal of Applied Physics Part 1-Regular Papers Short Notes and Review Papers, 1996, 35(3): 1983-1988.

106. Grill, W.M., W.M. Grill, and J.T. Mortimer, Stimulus waveforms for selective neural stimulation. Engineering in Medicine and Biology Magazine, IEEE, 1995, 14(4): 375-385.

107. Accornero, N., et al., Selective activation of peripheral-nerve fiber groups of different diameter by triangular shaped stimulus pulses. Journal of Physiology-London, 1977, 273(3): 539-560.

108. Havel, W.J., et al., Comparison of rectangular and damped sinusoidal dB/dt waveforms in magnetic stimulation. Magnetics, IEEE Transactions on, 1997, 33(5): 4269-4271.

109. Franco, S., Design with operational amplifiers and analog integrated circuits. 3rd ed. McGraw-Hill series in electrical and computer engineering. 2002, New York: McGrawHill, xiv, 658p.

110. Steele, J. and T. Green, Tame those versatile current source circuits. Elec. Des, 1992: $61-72$.

111. Smith, S., A.S. Sedra, and K.C. Smith, Microelectronic circuits, 4th ed. 1998, New York: Oxford University Press.

112. Microtechnology, A., Application note 19, 2004 online at: http://apex.cirrus.com/en/ products/apex/documents.html.

113. Microtechnology, A., Loop stability with reactive loads, in Application Note 38, 2007 online at: http://apex.cirrus.com/en/products/apex/documents.html.

114. Tian, M., et al., Striving for small-signal stability. Circuits and Devices Magazine, IEEE, 2001, 17(1): 31-41.

115. Engelhardt, M., Simulationsprogramm LT_SPICE (SwitcherCAD III).

116. Gray, P.R., Analysis and design of analog integrated circuits. 4th ed. 2001, New York: Wiley, xviii, 875p.

117. Williams, J., High speed amplifier techniques, Linear Technology Corporation. Application Note 47, 1991.

118. Williams, J., Power gain stages for monolithic amplifiers, Linear Technology Corporation. Application Note 18, March 1986.

119. Steele, J., Protect those expensive power Op amps. Electronic Design, 1991.

120. Stenzel, R.L., A new probe for measuring small electric fields in plasmas. Review of Scientific Instruments, 1991, 62: 130.

121. Glover, P.M. and R. Bowtell, Measurement of electric fields due to time-varying magnetic field gradients using dipole probes. Physics in Medicine and Biology, 2007, 52(17): 5119-5130.

122. Soonchil, L.E.E., L.E.E. Yongkwan, and Y.U. Insuk, Electric field in solenoids. Japanese Journal of Applied Physics, 2005, 44(7A): 5244-5248.

123. Kandel, E.R., J.H. Schwartz, and T.M. Jessell, Principles of neural science. 4th ed. 2000, New York: McGraw-Hill, Health Professions Division, xli, 1414p.

124. Willms, A.R., Neurofit: software for fitting Hodgkin-Huxley models to voltage-clamp data. Journal of Neuroscience Methods, 2002. 121: 139-150.

125. Horch, K.W. and G.S. Dhillon, Neuroprosthetics theory and practice. Series on bioengineering and biomedical engineering. 2004, River Edge, NJ: World Scientific, xxv, 1261p.

126. Lertmanorat, Z. and D.M. Durand, A novel electrode array for diameter-dependent control of axonal excitability: a Simulation study. Biomedical Engineering, IEEE Transactions on 2004, 51(7): 1242-50.

127. Glantz, R.M. and T. Viancour, Integrative properties of crayfish medial giant neuron: steady-state model. J Neurophysiol, 1983, 50(5): 1122-1142. 
128. Watanabe, A. and H. Grundfest, Impulse propagation at the septal and commissural junctions of crayfish lateral giant axons. The Journal of General Physiology, 1961, 45(2): 267-308.

129. Mellon, D. and C. Kaars, Role of regional cellular geometry in conduction of excitation along a sensory neuron. Journal of Neurophysiology, 1974, 37(6): 1228-1238.

130. Haynes, L.W. and G.A. Kerkut, Response time constants in snail neurones. Cellular and Molecular Life Sciences (CMLS), 1977, 33(11): 1482-1483.

131. Ratte, S. and R. Chase, Synapse distribution of olfactory interneurons in the procerebrum of the snail Helix aspersa. The Journal of Comparative Neurology, 2000, 417(3): 366-384.

132. Gillary, H.L., Electrical responses from the eye ofHelix to photic stimulation and simultaneous electrical stimulation of the optic nerve. Vision Research, 1970, 10(10): 977-991.

133. Antkowiak, T. and R. Chase, Sensory innervation of the ovotestis in the snail Helix aspersa. Journal of Experimental Biology, 2003, 206(22): 3913-3921.

134. Clarac, F. and E. Pearlstein, Invertebrate preparations and their contribution to neurobiology in the second half of the 20th century. Brain Research Reviews, 2007, 54(1): $113-161$.

135. William, R.K., Opportunities afforded by the study of unmyelinated nerves in skin and other organs. Muscle and Nerve, 2004, 29(6): 756-767.

136. Kerkut, G.A., et al., Mapping of nerve cells in the suboesophageal ganglia of Helix aspersa. Comparative Biochemistry and Physiology A, 1975, 50(1A): 1-25.

137. Prescott, S. and R. Chase, Two types of plasticity in the tentacle withdrawal reflex of Helix aspersa are dissociated by tissue location and response measure. Journal of Comparative Physiology A: Sensory, Neural, and Behavioral Physiology, 1996, 179(3): 407-414.

138. Wyttenbach, R.A., et al., Crawdad a CD-ROM lab manual for neurophysiology. 1999, Sinauer Associates.

139. Olivo, R.F., An online lab manual for neurophysiology. Journal of Undergraduate Neuroscience Education, 2003, 2: A16-A22.

140. Cattaert, D. and D. Le Ray, Adaptive motor control in crayfish. Progress in Neurobiology, 2001, 63(2): 199-240.

141. Jacklet, J.W., Neuronal and cellular oscillators. Cellular clocks. 1989, New York: Marcel Dekker, xiii, 553p.

142. Kettenmann, H. and R. Grantyn, Practical electrophysiological methods: a guide for in vitro studies in vertebrate neurobiology. 1992. New York: Wiley-Liss, xviii, 449p.

143. Stamford, J.A., Monitoring neuronal activity: a practical approach. Practical approach series. 1992. Oxford; New York: IRL Press at Oxford University Press, xxii, 294p.

144. Purves, R.D., Microelectrode methods for intracellular recording and ionophoresis. Biological techniques series. 1981. London; New York: Academic Press, x, 146p.

145. Dempster, J. and J. Dempster, The laboratory computer : a practical guide for physiologists and neuroscientists. Biological techniques series. 2001. San Diego: Academic Press, xii, 354 .

146. Land, B.R., R.A. Wyttenbach, and B.R. Johnson, Tools for physiology labs: an inexpensive high-performance amplifier and electrode for extracellular recording. Journal of Neuroscience Methods, 2001, 106(1): 47-55.

147. Guclu, B., Low-cost computer-controlled current stimulator for the student laboratory. Advances in Physiology Education, 2007, 31(2): 223-231.

148. Wagenaar, D.A. and S.M. Potter, A versatile all-channel stimulator for electrode arrays, with real-time control. Journal of Neural Engineering, 2004, 1(1): 39-45.

149. Land, B.R., et al., Tools for physiology labs: inexpensive equipment for physiological stimulation. Journal of Undergraduate Neuroscience Education, 2004, 3: A30-A35.

150. Eagar, D., Application Note 87. Linear Technology. 
151. Lott, G., Hybridizing cellular and behavioral neurobiology with modern engineering tools: microelectronics, microfabricated devices, and software solutions for physiology. 2007, New York: Cornell University.

152. Lidierth, M., SigTool: a matlab-based environment for sharing laboratory-developed software to analyze biological signals. Journal of Neuroscience Methods, 2009, 178: 188-196.

153. Quiroga, R.Q., Z. Nadasdy, and Y. Ben-Shaul, Unsupervised spike detection and sorting with wavelets and superparamagnetic clustering. 2004. Cambridge, MA: MIT Press, pp. 1661-1687.

154. Fitzgerald, J., Recording with microchannel electrodes in a noisy environment. 2008.

155. Basham, E., W. Liu, and Y. Zhi, A System for quantitative analysis of magnetic neural stimulation. 2008, in review.

156. Hsu, K.H. and D.M. Durand, A 3-D differential coil design for localized magnetic stimulation. Biomedical Engineering, IEEE Transactions on, 2001, 48(10).

157. Maccabee, P.J., et al., Magnetic coil stimulation of straight and bent amphibian and mammalian peripheral nerve in vitro: locus of excitation. Journal of Physiology (London), 1993, 460: 201-219.

158. Mulloney, B., N. Tschuluun, and W. M. Hall, Architectonics of crayfish ganglia. Microscopy Research and Technique, 2003, 60: 253-265.

159. Prescott, S. A., N. Gill, and R. Chase, Neural Circuit Mediating Tentacle Withdrawal in Helix aspersa, With Specific Reference to the Competence of the Motor Neuron C3. Journal of Neurophysiol, 1997, 78: 2951-2965, December 1, 1997. 